\title{
Prótesis de rodilla externa mecatrónica
}

\author{
F. VALENCIA ${ }^{1}$, X. LIMA ${ }^{1}$, D.OJEDA ${ }^{2}$, D. ORTIZ ${ }^{1}$ \\ ${ }^{1}$ Department of engineering in mechatronics, Universidad Técnica Del Norte, Ibarra-Ecuador \\ ${ }^{2}$ Prometeo Investigador SENESCYT, Universidad Técnica del Norte, Ibarra-Ecuador
}

\begin{abstract}
Resumen
Se diseña un mecanismo de articulación de rodilla para una prótesis externa, es decir el prototipo puede ser usado en personas con amputaciones del tipo transfemoral, con el fin de contribuir a la sociedad al brindar soluciones a favor de las personas con capacidades especiales. Se toma en consideración los aspectos más relevantes para el desarrollo de la marcha humana protésica normal como son: nivel de actividad fisica, medidas antropométricas, materiales adecuados para la construcción, estabilidad y confort entre otros. Luego de realizar un análisis extensivo y proceso de diseño y rediseño se construyó un prototipo funcional, que en el futuro podrá ser probado en personas que asi lo requieran.
\end{abstract}

Palabras clave: Antropometría, prótesis, amputación, biomecánica, transfemoral.

\begin{abstract}
The research aims the mechanical development prototype of a mechatronic external knee prosthesis for transfemoral amputation type. The prototype in process of development is considered to be an intelligent prosthesis since it is able to be to be controlled by signals from the biological body. The designing of the process of the knee mechanism is detailed in this document. The aspects considered in development are: the mechanical relationship with the human body and the human motion analysis, as principal function of the prosthesis that enables to determine the forces involved in the development of the motion, the angles of knee displacement and motion speed. The strain distribution under different compressive load conditions were simulated by using the finite element method.
\end{abstract}

Keywords: Anthropometry, prosthesis, amputation, biomechanics, transfemoral.

\section{Introducción}

De acuerdo con el Consejo Nacional de Discapacidades del Ecuador (CONADIS). En ecuador 397233 personas tiene algún tipo de discapacidad y aproximadamente 193905 personas tienen algún tipo de discapacidad física en todo el país. De esta cifra 4620 personas tienen discapacidad física en la provincia de Imbabura[1].

Correspondencia:

Correspondencia: F. Valencia

E-mail: fvvalencia@utn.edu.ec
La "Fundación prótesis para la vida" cuenta con un estimado de 201 pacientes protetizados para una amputación del tipo transfemoral, que provienen de distintas partes del Ecuador.

En la actualidad el desarrollo de tecnología para mejorar la calidad de vida es cada vez más importante, así también los diferentes tipos de prótesis se han rediseñado para proporcionar a la persona protetizada, la posibilidad de realizar una marcha humana controlada y estabilidad cuando se encuentra de pie.

El análisis de la marcha humana contribuye en la determinación de los principales paráme- 
tros del prototipo tales como: peso, fuerza, equilibrio, determinación del material.

La Construcción de un mecanismo de articulación de rodilla para prototipo de prótesis externa mecatrónica contribuye en el desarrollo de una prótesis inteligente capaz de ser controlada por señales biológicas, proporcionando un mejor control de la prótesis durante las diversas fases de la marcha.

El diseño final propuesto se obtiene luego de realizar tres bocetos de prótesis, establecer factores de selección adecuados y cambios respectivos con el fin de desarrollar una prótesis mecatrónica en la Universidad Técnica del Norte.

Para una primera fase de desarrollo del prototipo, su principal función es la de restablecer la capacidad de bipedestación en personas, es decir comenzar a usar una prótesis definitiva esto implica realizar la marcha a una velocidad baja en superficies planas sin obstáculos como bordillos o escaleras.

\section{Materiales y método}

\section{Prótesis de rodilla externa}

Una prótesis de rodilla externa transfemoral como su nombre lo indica es una prótesis exclusiva para una persona que ha sufrido una amputación a nivel transfemoral (Fig. 1) y son conocidas generalmente como prótesis por encima de la rodilla.

Las prótesis mecánicas utilizan la fricción entre componentes para tener un mayor control al realizar la marcha humana y pueden clasificarse por su constitución monocéntrica (Fig. 2a) y policéntricas (Fig. 2b).

El término "inteligente" en las prótesis de miembros inferiores indica que la prótesis es capaz de cambiar su respuesta al caminar. Este concepto surgió una vez que la electrónica se aplicó al campo prótesis por el profesor Flores y sus colaboradores en el MIT, durante la década de los 70 [2].

Las prótesis inteligentes (Fig. 3) utilizan sistemas de control para las diversas fases de la marcha con el fin de reproducir una marcha mas natural.

En las prótesis de pierna existen aún algunos problemas, por ejemplo, los amputados que utilizan prótesis mecánicas requieren entre 10-60\% más energía metabólica que las personas con sus extremidades inferiores completas, dependiendo de la velocidad de caminado, el estado físico de la persona, la causa de la amputación,
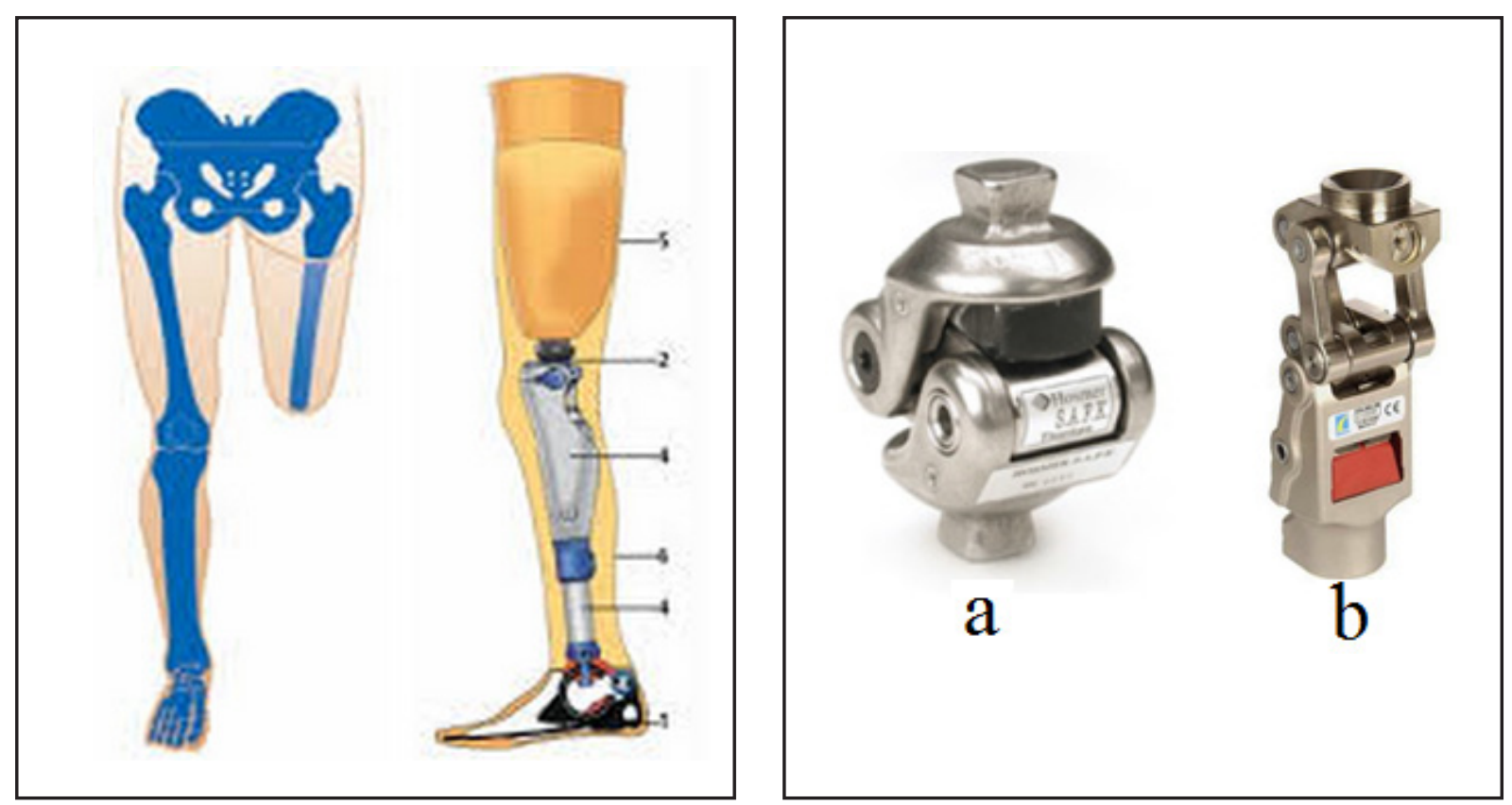

Figura 1 (izquierda). Prótesis de rodilla transfemoral.

Figura 2 (derecha). Prótesis mecánicas a) Monocéntrica b) Policéntrica. 
el nivel de amputación, y las características de la prótesis. Además, los amputados caminan entre $10-40 \%$ más lento que las personas intactas [3].

La creación de una prótesis inteligente proporciona al paciente protetizado un mayor control al realizar una marcha humana, además de reducir la energía metabólica utilizada al caminar, debido a que la fuerza motora va a ser proporcionada por el mecanismo de accionamiento de la rodilla.

\section{Características de la prótesis}

Para determinar las características del prototipo es indispensable analizar el movimiento de flexo-extensión que realiza la rodilla, y seleccionar un sujeto de estudio.

La flexo-extensión es el movimiento principal de la rodilla. Su amplitud se mide a partir de la posición de referencia definida de la siguiente manera: el eje de la pierna se sitúa en la prolongación del eje del muslo (Fig. 4, pierna derecha).

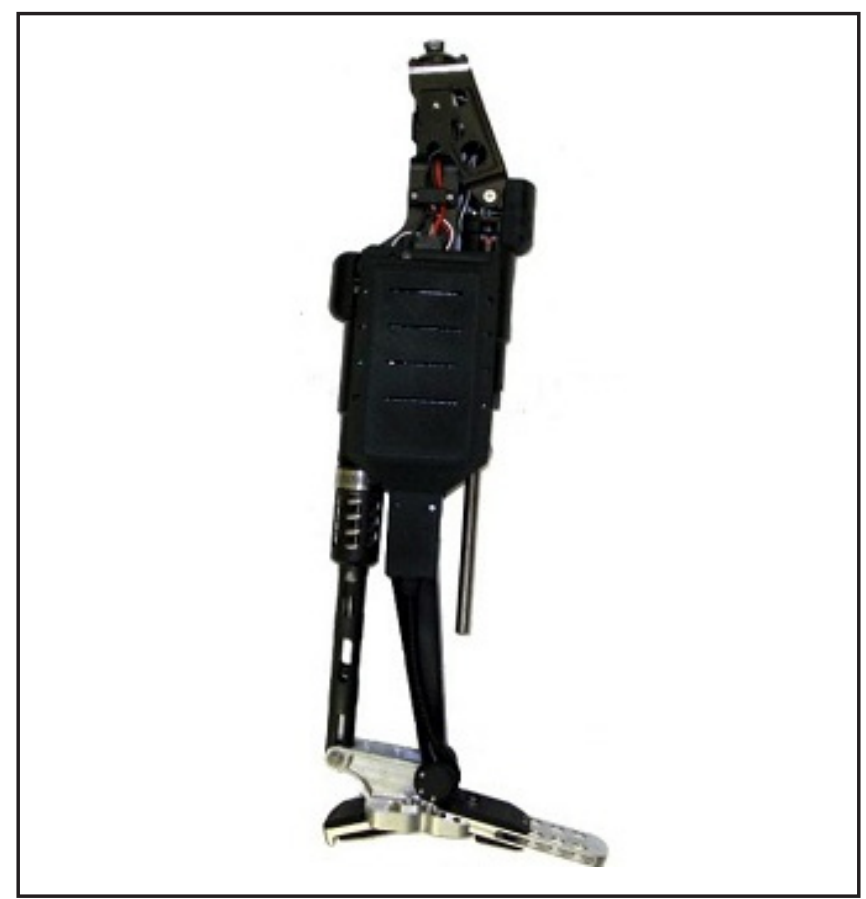

Figura 3. Prótesis inteligente.

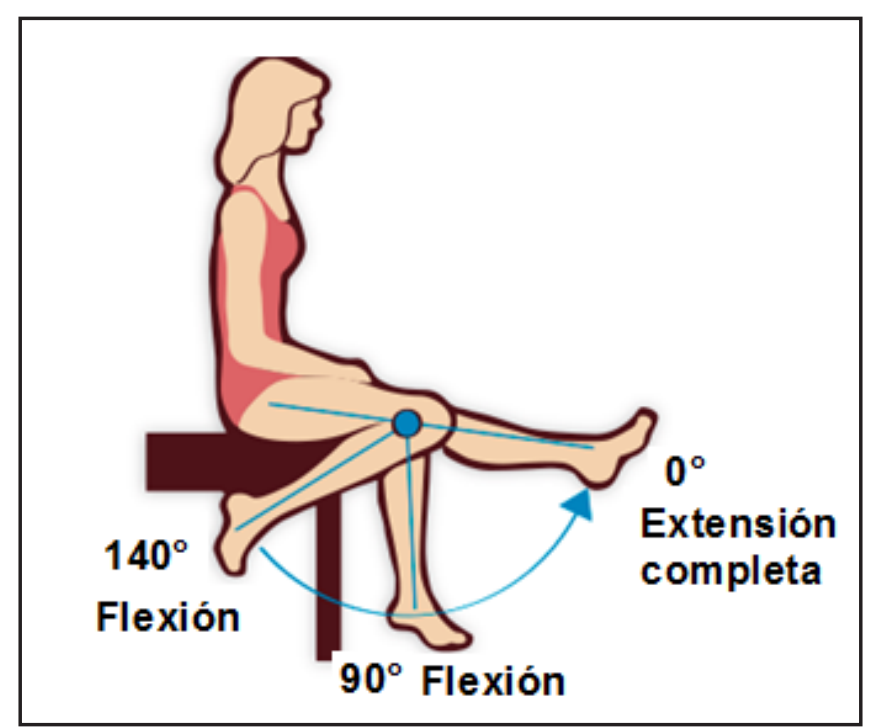

Figura 4. Flexión de la rodilla. 
De perfil, el eje del fémur continúa sin ninguna angulación. Con el eje del esqueleto de la pierna. En la posición de referencia, el miembro inferior posee su máxima longitud. [4]

Murray et al.(1964) midieron con un electrogoniómetro el rango de movimento en el plano sagital durante la marcha. Se observó la extensión completa o casi completa en el inicio de la fase $(0 \%$ del ciclo) con el contacto del talón, y al final de la fase portante antes de despegar los dedos (alrededor del 60\% del ciclo) se observa la máxima flexión(aproximadamente $60^{\circ}$ ) durante la mitad de la fase de oscilación.[5]

Para la actividad física que implica una marcha humana es necesario $60^{\circ}$ de flexión en la rodilla, el prototipo cuenta con $80^{\circ}$ de flexión suficientes para la ejecución de la misma.

Para determinar las dimensiones del prototipo, se toma en consideración los segmentos que constituyen muslo, pie y pierna en características de longitud y masa a partir de estudios antropométricos.

Se selecciona una persona de estudio de género masculino y adulta con una estatura de $1,72 \mathrm{~m}$ y $65 \mathrm{~kg}$ de masa para aplicar los estudios antropométricos que constan en la tabla I.

Los datos de la tabla I son estudios de una población de América del Norte, presentan variaciones de acuerdo a la población del Ecuador, sin embargo nos proporciona valores cercanos. Para la tabla I ( $\mathrm{H}$ y M) corresponden a la estatura y masa de la persona respectivamente.
Los resultados de las dimensiones del prototipo de acuerdo al sujeto de estudio se muestran en la tabla II, estos valores son referenciales y se diseña el mecanismo con valores inferiores para evitar dificultades al usar el prototipo.

El ancho de la pierna y muslo es difícil determinar debido a que esta consta de músculos y tejido adiposo, es por ello que se toma en consideración $105 \mathrm{~mm}$ de ancho como máximo para tener un prototipo estético.

El resumen de las caracteísticas de la prótesis se muestra en la tabla III:

\section{Selección de materiales}

Los materiales que constituyen la prótesis son elegidos de acuerdo a las cargas que soporta la prótesis y es a la vez dependiente del nivel de actividad del paciente.

No existe un material adecuado para todos los pacientes protetizados, sino más bien se debe tomar en consideración las aspiraciones que tiene el paciente respecto a la prótesis y su estilo de vida.

Es necesario que el prototipo soporte el peso del cuerpo humano, además de ser lo más liviano posible. Por tal motivo se usa generalmente en prótesis comerciales los siguientes materiales: aleaciones de aluminio, titanio, fibra de carbón, acero y polímeros.

Se hace uso de un software que contiene una amplia variedad de materiales comerciales con

\begin{tabular}{|l|l|l|l|}
\hline & Muslo & Pierna & Pie \\
\hline Longitud & $0,245 \mathrm{H}$ & $0,246 \mathrm{H}$ & $0,152 \mathrm{H}$ \\
\hline Masa & $0,1 \mathrm{M}$ & $0,0465 \mathrm{M}$ & $0,0145 \mathrm{M}$ \\
\hline
\end{tabular}

Tabla 1. Datos antropométricos de los segmentos corporales de la extremidad inferior[6].

\begin{tabular}{|l|l|}
\hline Masa & $\mathbf{6 5} \mathbf{~ k g}$ \\
\hline Longitud & $1,72 \mathrm{~m}$ \\
\hline Dimensión de la pierna & $0,42 \mathrm{~m}$ \\
\hline Masa de la pierna & $3,0225 \mathrm{~kg}$ \\
\hline
\end{tabular}

Tabla 2. Dimensiones de la pierna. 
sus propiedades y facilita la selección del material más adecuado, haciendo uso de dichas propiedades al establecer valores máximos y mínimos, como se muestra en la tabla IV.

Los materiales que cumplen con las condiciones de la tabla IV son aleaciones de titano, acero aleado, carburo de silicio, alúmina, aleaciones de zinc y aleaciones de aluminio, siendo el carburo de silicio un material que no se ha probado en prótesis al igual que la alúmina, sino más bien en la elaboración de herramientas entre otras aplicaciones, son eliminados de la lista con ayuda del software.

Para seleccionar el material más adecuado se hace uso de tablas comparativas de acuerdo con las relaciones de propiedades entre materiales, como se muestra en la figura 5 y figura 6.

Las gráficas de materiales de las fig. 5 y fig. 6 se resumen en la tabla $\mathrm{V}$ en orden ascendente de acuerdo a lo siguiente: el material con mejores características se ubica en la posición 1 y a continuación los materiales restantes toman sus posiciones, como se observa en la tabla v.
De acuerdo a los parámetros antes mencionados, el material más adecuado es seleccionado de la tabla $\mathrm{V}$ y es el aluminio por su buena relación límite elástico - densidad y relación precio - densidad.

En el Ecuador podemos encontrar aleaciones de aluminio 6061 y 7075 T6, la primera aleación se usa en la fabricación de bicicletas y marcos de ventanas entre otras aplicaciones, mientras que la aleación 7075 T6, también llamada aluminio Prodax se utiliza para matricería por sus buenas características para mecanizar.

Es por ello que se decide utilizar aluminio 7075 T6 en la fabricación del mecanismo; sus propiedades se muestran en la tabla VI.

\section{Resultados}

\section{Diseño de la articulación}

El modelo del mecanismo cuenta con un espacio interior en el cual se ubica un actuador lineal que genera la fuerza motora para la articulación de la rodilla. El mecanismo completo

\begin{tabular}{|l|c|}
\hline Característica & Descripción \\
\hline Tipo de rodilla & Monocéntrica \\
\hline Nivel de actividad & $\begin{array}{c}\text { Nivel de actividad física } 1 \\
\text { (espacios interiores sin obstáculos) }\end{array}$ \\
\hline Útil para amputación & Transfemoral \\
\hline Flexión y extensión & $80^{\circ}$ \\
\hline Dimensiones & $\begin{array}{c}\text { Ancho: } 0.105 \mathrm{~m} \\
\text { Largo: } 0.420 \mathrm{~m}\end{array}$ \\
\hline Peso máximo & $3 \mathrm{~kg}$ \\
\hline Peso del paciente & $1 . \mathrm{kg}$ \\
\hline
\end{tabular}

Tabla 3. Cacterísticas del prototipo de rodilla.

\begin{tabular}{|l|c|c|c|}
\hline Propiedad & Valor mínimo & Valor máximo & Relación \\
\hline Densidad & 2600 & 8000 & $\mathrm{~kg} / \mathrm{m} 3$ \\
\hline Precio & 1 & 40 & $\mathrm{USD} / \mathrm{kg}$ \\
\hline Límite elástico & 100 & 500 & $\mathrm{MPa}$ \\
\hline
\end{tabular}

Tabla 4. Propiedades de un material para prótesis. 


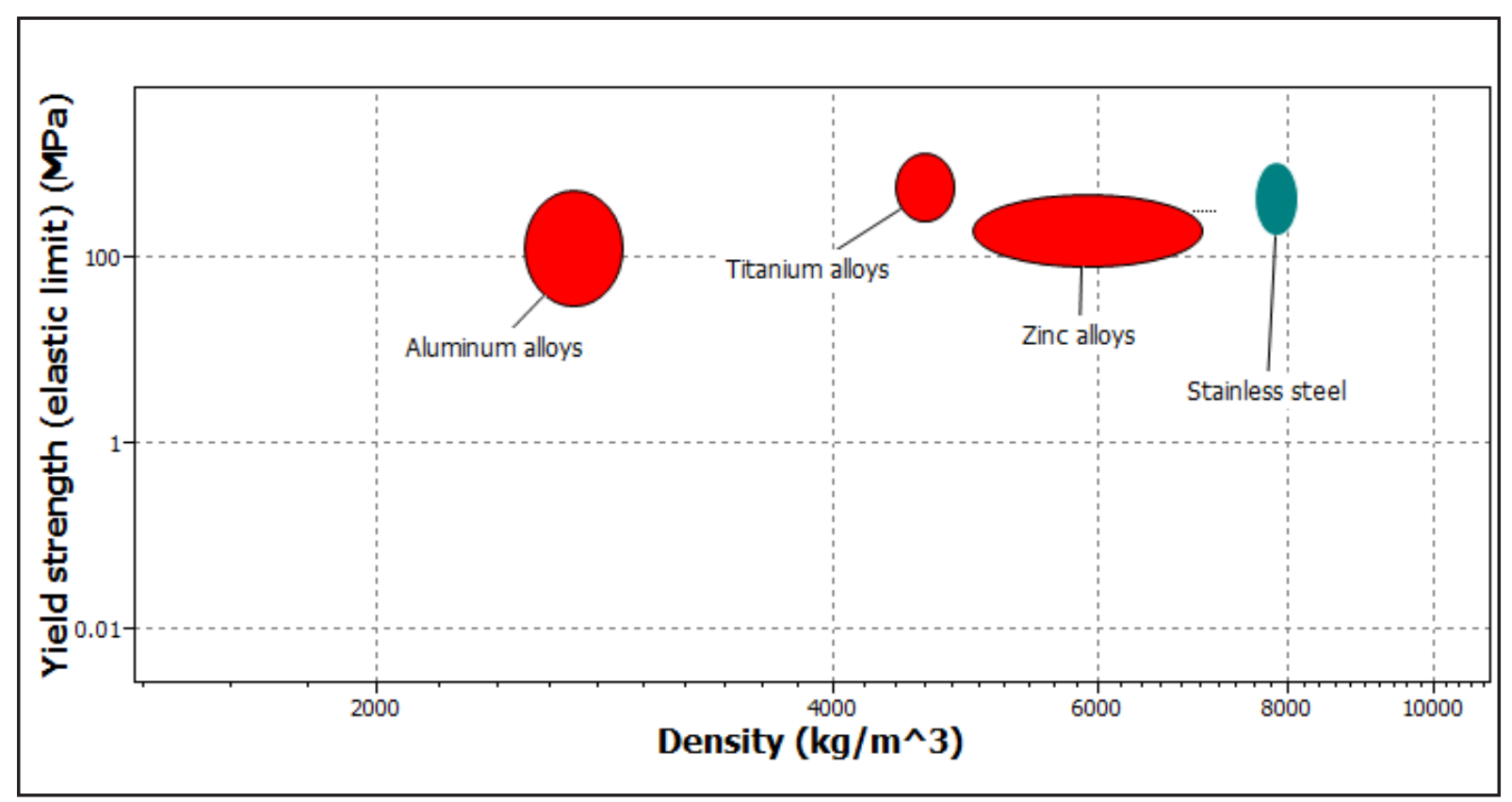

Figura 5. Gráfica de materiales Límite elástico - densidad.

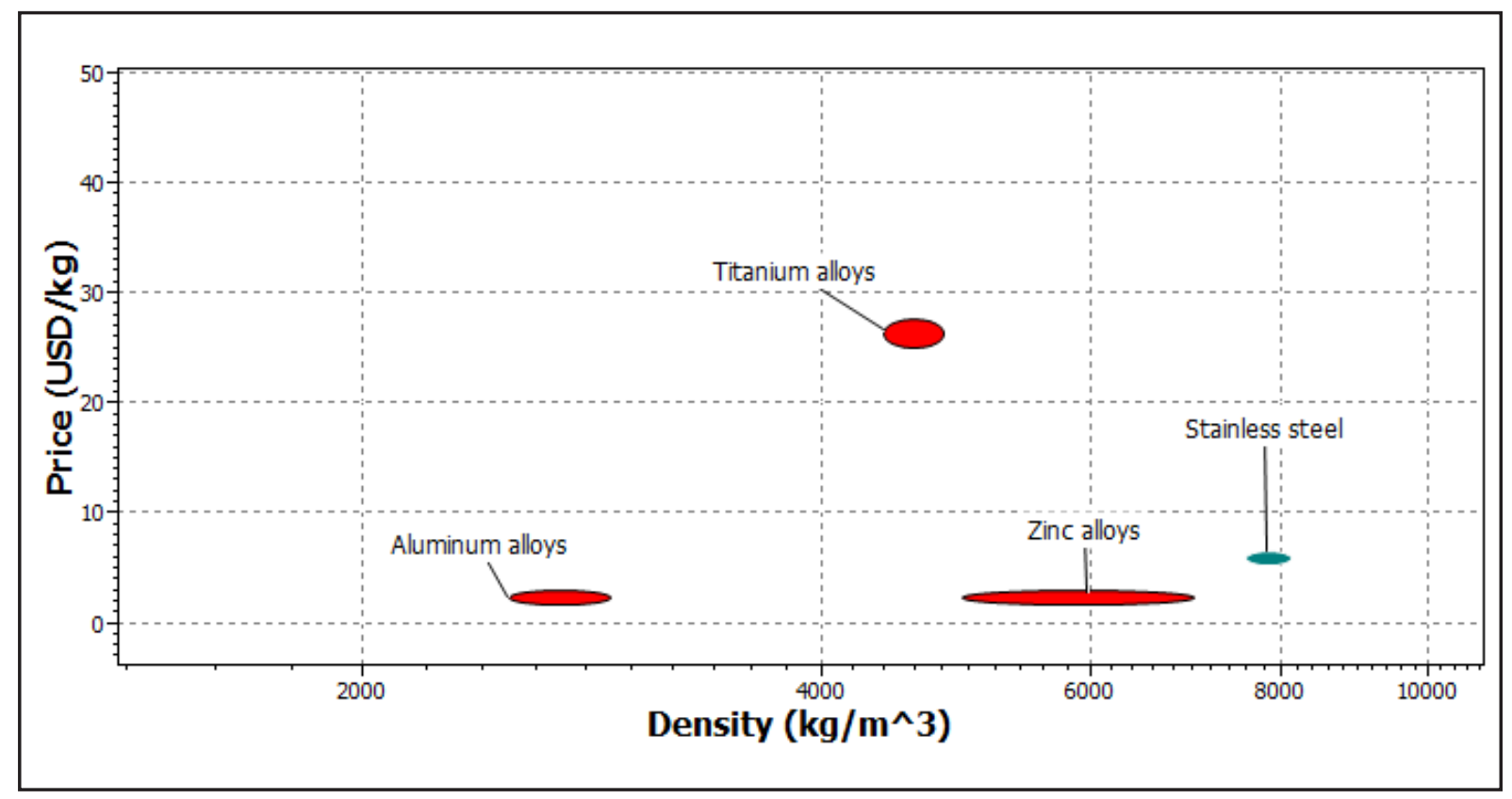

Figura 6. Gráfica de materiales Precio - densidad.

\begin{tabular}{|c|c|c|}
\hline Material & $\begin{array}{c}\text { Relación } \\
\text { límite elástico-densidad }\end{array}$ & $\begin{array}{c}\text { Relación } \\
\text { precio - densidad }\end{array}$ \\
\hline Aleaciones de Aluminio & 1 & 1 \\
\hline Aleaciones de titanio & 2 & 4 \\
\hline Aleaciones de Zinc & 3 & 2 \\
\hline Acero inoxidable & 4 & 3 \\
\hline
\end{tabular}

Tabla 5. Selección del material. 
se puede observar en la fig. 8 .

El mecanismo consta de varias partes, una parte móvil que efectúa el movimiento de flexión y una parte fijada a la extremidad del pie a través de un pilón (pilar de metal que unirá la rodilla al pie protésico) y acoples respectivamente, además cuenta con pasadores que per- miten el movimiento rotacional de la articulación y la transmisión de fuerza del actuador al mecanismo, como se muestra en la figura 8.

A continuación se detallan individualmente las partes constitutivas del mecanismo de rodilla monocéntrica mostrada en la fig. 8 y su función en la tabla VII.

\begin{tabular}{|l|c|}
\hline Propiedades & Valor \\
\hline Módulo elástico & $72000 \mathrm{MPa}$ \\
\hline Coeficiente de Poisson & 0.33 \\
\hline Límite elástico & 505 \\
\hline Límite de tracción & $570 \mathrm{MPa}$ \\
\hline Densidad & $1 . \mathrm{m}^{3}$ \\
\hline
\end{tabular}

Tabla 6. Propiedades del aluminio 7075 T6 [8].

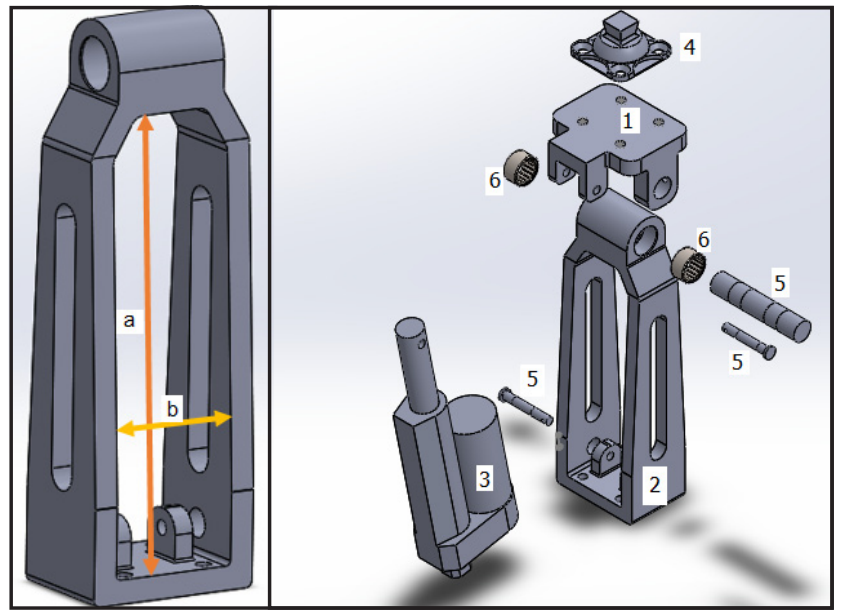

Figura 7 (izquierda). Espacio del actuador a) $18,5 \mathrm{~cm} \mathrm{b)} 5,2 \mathrm{~cm}$. Figura 8 (derecha). Mecanismo de rodilla.

\begin{tabular}{|c|c|c|}
\hline Nro & Nombre & Función \\
\hline 1 & Parte móvil & $\begin{array}{l}\text { Referenciar el ángulo de la rodilla de flexión de } \\
\text { acuerdo a la acción del cilindro neumático. }\end{array}$ \\
\hline 2 & Estructura base & $\begin{array}{l}\text { Contener el actuador lineal y proveer soporte a los } \\
\text { componentes restantes de la prótesis como el pie. }\end{array}$ \\
\hline 3 & Actuador lineal & $\begin{array}{l}\text { Proveer la fuerza motora para la flexión de la } \\
\text { rodilla durante las fases de un ciclo de marcha. }\end{array}$ \\
\hline 4 & $\begin{array}{l}\text { Acople superior de } \\
\text { pirámide }\end{array}$ & $\begin{array}{c}\text { Sujeción entre el mecanismo y la cuenca de la } \\
\text { persona protetizada. }\end{array}$ \\
\hline 5 & Pasadores & Servir de guía en las articulaciones. \\
\hline 6 & Rodamientos & $\begin{array}{l}\text { Reducir el contacto entre los pasadores y el } \\
\text { mecanismo. }\end{array}$ \\
\hline
\end{tabular}

Tabla 7. Descripción y función de las partes del mecanismo. 
La forma de cada componente se ajusta a los movimientos requeridos con el fin de evitar interferencia entre componentes así como sus dimensiones.

Los elementos que se fabrican son la estructura base, la parte móvil y los pasadores, los elementos constitutivos restantes tales como cuenca o encaje, adaptadores de cuenca, acople de pirámides, pilón entre otros son seleccionados debido a que son elementos estándares de uso en todas las prótesis.

\section{Selección del actuador}

Para la selección del actuador se estudia el proceso de marcha humana a fin de determinar la fuerza necesaria del actuador durante un ciclo de la misma. (ver figura 9)

El ciclo de marcha normal inicia con el contacto del talón con la superficie del suelo y termina con el siguiente contacto del mismo talón [9] (ver figura 10)
La cantidad relativa de tiempo gastado durante cada fase del ciclo de la marcha según el Instituto de Biomecánica de Valencia, a una velocidad normal de 100 a 115 pasos por minuto es:

1. Fase de apoyo: $60 \%$ del ciclo

2. Fase de balanceo: $40 \%$ del ciclo

3. Doble apoyo: $20 \%$ del ciclo.

$\mathrm{Al}$ analizar el ciclo de marcha de acuerdo a la figura 9, se puede observar que la fase más importante se produce en la fase de apoyo de un solo soporte, ya que durante esta fase, el cuerpo es soportado en una sola pierna, así entonces en esta fase el mecanismo de la rodilla soporta la mayor carga.

Los dos principales momentos (Fig. 11) que actúan alrededor del centro del movimiento de la articulación son: El momento de flexión sobre la pierna producto del peso corporal y su brazo de palanca "a" y el momento de extensión de

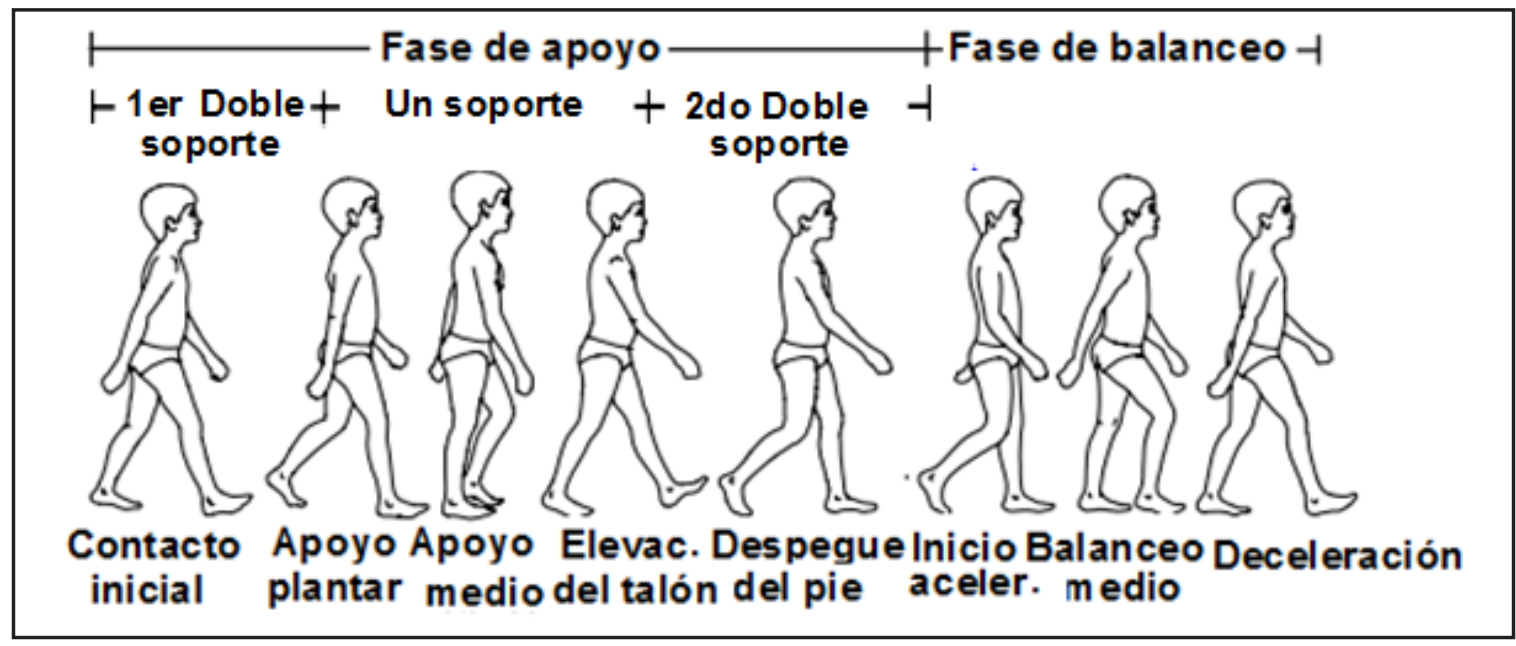

Figura 9. Marcha humana[9].

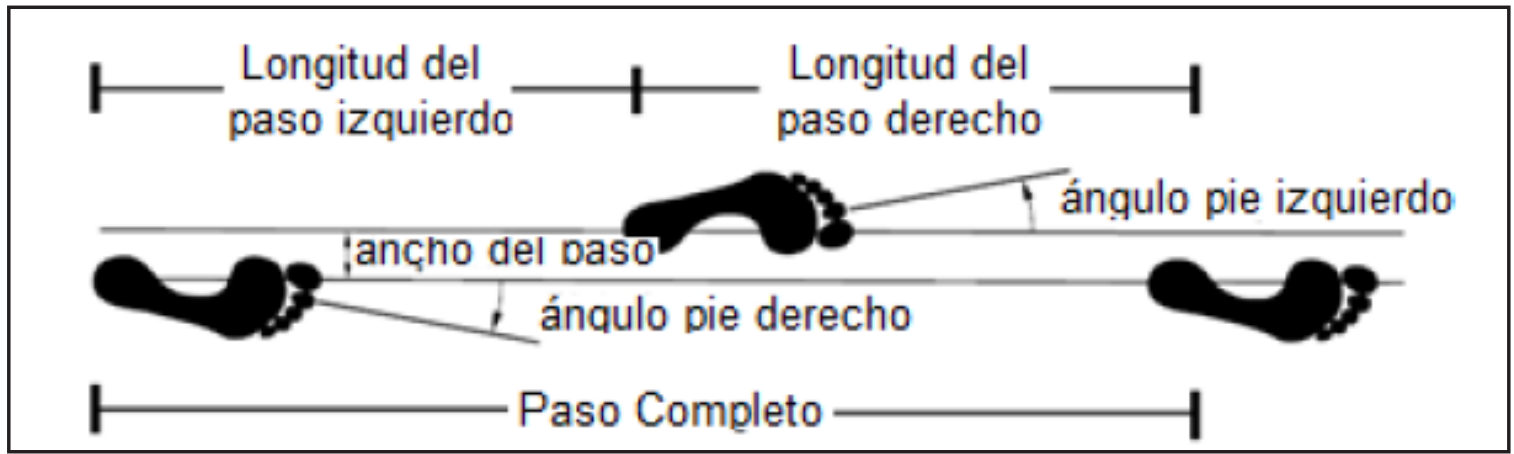

Figura 10. Ciclo de la marcha[9]. 
contraposición producto de la fuerza del músculo cuádriceps a través del tendón rotuliano y su brazo de palana "b" [10].

El torque es un producto de la fuerza muscular principal aceleradora de la región corporal y la distancia perpendicular de la fuerza desde el centro del movimiento de la articulación[10].

Ecuación 1: Torque en la articulación de la rodilla [10]

$$
\mathrm{T}=\mathrm{F} \cdot \mathrm{d}
$$

Para el cálculo de la fuerza en el modelo de prótesis se toma en cuenta la distancia perpendicular a la fuerza aplicada en la parte móvil del dispositivo, como se muestra en la fig 12 .

Durante la marcha normal los momentos generados en la rodilla debido a la reacción del pie de apoyo con la superficie del suelo varían a través de todas las fases del ciclo de la marcha, es decir que los momentos articulares que se generan por parte de los músculos varían en todo el ciclo de marcha.

Las figuras 13 y 14 proporcionan una gráfica de los ángulos y momentos articulares que se generan en la rodilla durante un ciclo de marcha, expresada en porcentajes.

A través del análisis de la figura 14 es posible determinar los puntos críticos o de mayor esfuerzo en función del torque que se genera por el peso de la persona y a través de la figura 13 es posible determinar el ángulo en el que se encuentra la rodilla cuando ocurre el mayor esfuerzo. Esto es posible ya que las gráficas se encuentran en función del ciclo de marcha expresado en porcentajes.

Los mayores momentos articulares, de acuerdo a la fig 14, se encuentra en la fase de apoyo, es decir dentro del $60 \%$ inicial del ciclo de marcha y durante la fase de oscilación disminuyen, esto se debe a que durante la fase de apoyo la rodilla está bajo la compresión ejercida por el peso del cuerpo, mientras que en la fase de balanceo $u$ oscilación solamente el peso de la pierna ejerce sobre la rodilla una fuerza de tracción.

- $\quad$ Fase de apoyo. > Una sola pierna recibe el peso del cuerpo (compresión).

- Fase de oscilación. > La gravedad influye sobre el peso de la pierna (tracción).
Así entonces en función de la masa de la persona protetizada se obtiene el momento necesario durante la marcha de la figura 14 y en función de dicho momento articular se calcula la fuerza necesaria del actuador.

Es evidente que los momentos máximos se presentan los puntos 1, 2, 3 y 4 como se observa en las figuras 13 y 14 corresponden a 2.86, 15.71, 0 y 12.86 grados de flexión en la articulación de la rodilla.

Los momentos articulares están en función del peso de la persona en estudio como se muestra en la figura 14 así entonces, la ecuación 2 se utiliza para los puntos 1 y 2 , la ecuación 3 se utiliza para el punto 3 y la ecuación 4 se utiliza para el punto 4, ecuaciones que se obtienen del análisis de la figura 13.

Ecuación 2: Momento articular máximo para el punto 1 y 2 de análisis

$$
\mathrm{T}=0,4 \mathrm{Nm} / \mathrm{kg}
$$

Ecuación 3: Momento articular para el punto 3 de análisis

$$
\mathrm{T}=0,3 \mathrm{Nm} / \mathrm{kg}
$$

Ecuación 4: Momento articular para el punto 4 de análisis

\section{$\mathrm{T}=0,25 \mathrm{Nm} / \mathrm{kg}$}

La tabla VIII muestra los resultados obtenidos de las ecuaciones 2, 3 y 4 con los datos de la persona en estudio.

La fuerza necesaria del actuador se determina a partir del análisis de momentos articulares en los puntos 1, 2, 3 y 4 de la sección anterior, como se muestra en la tabla VIII y del brazo de palanca dependiente de la geometría del modelo de rodilla como se muestra en la figura 15 para un ángulo de flexión de $0^{\circ}$.

Los segmentos $\mathrm{BC}$ y CA de la figura 15 permanecen constantes, mientras que el segmento BA varía según el ángulo de flexión en la rodilla, el ángulo ACB es 100 grados para una flexión de 0 grados en la rodilla y el brazo de palanca que determine el torque empieza en $\mathrm{C}$ y es perpendicular al segmento BA.

Es importante considerar que la dirección de aplicación de la fuerza por parte del actuador es la misma del segmento BA, que varía durante los 


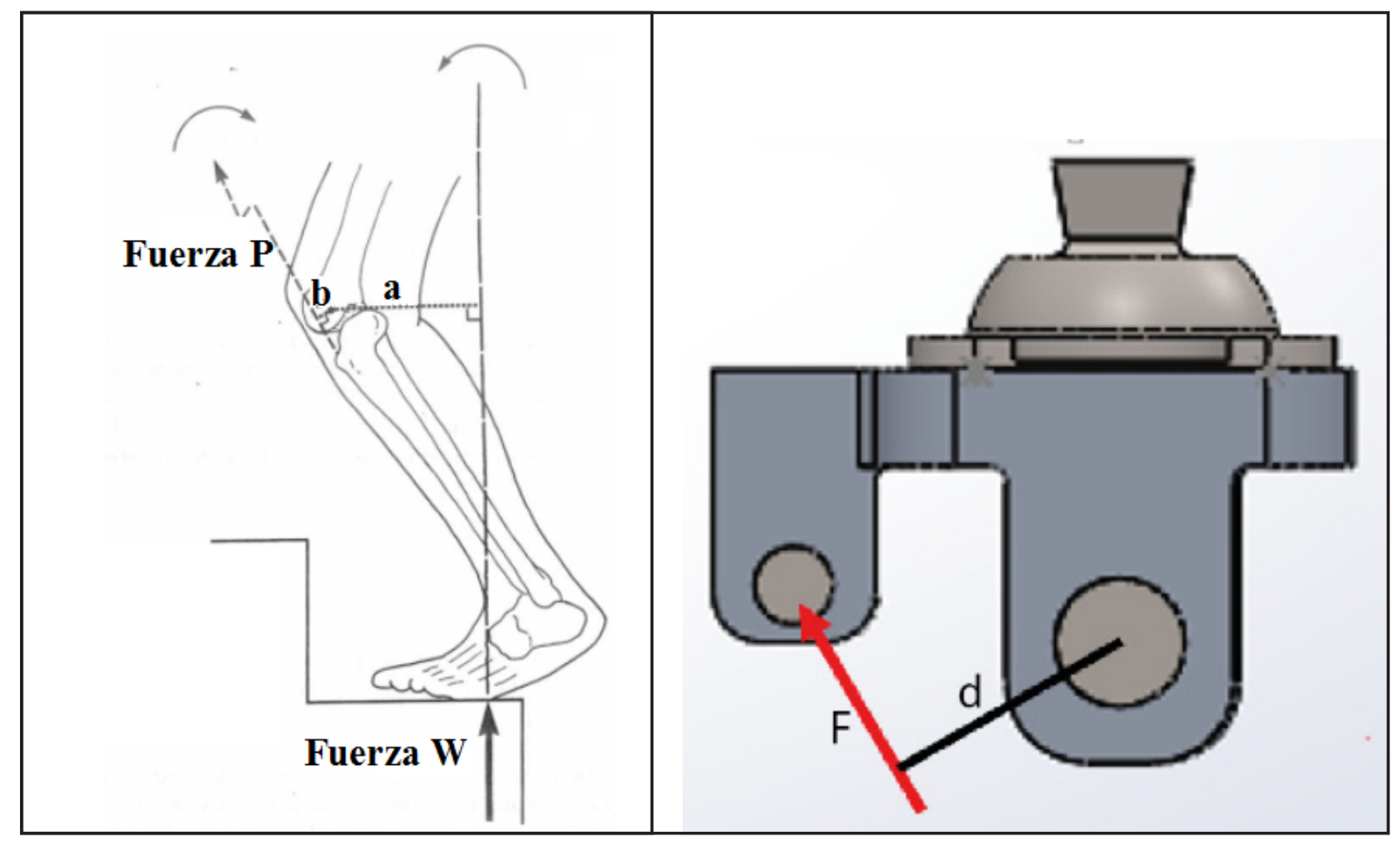

Figura 11 (izquierda). Momentos articulares [10].

Figura 12 (derecha). Articulación de la rodilla a 0 grados de flexión.

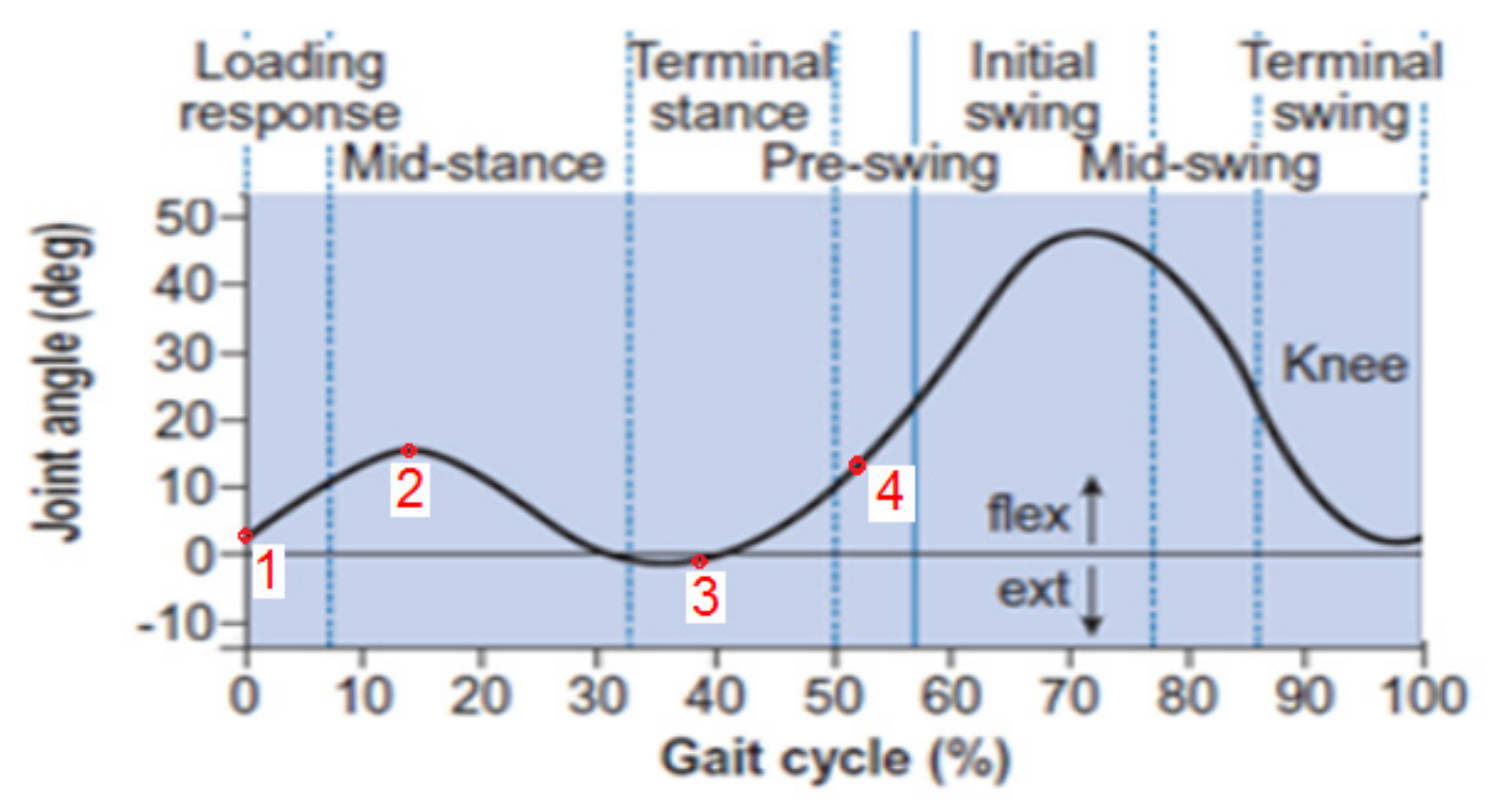

Figura 13. Ángulos de la articulación durante la marcha[7]. 


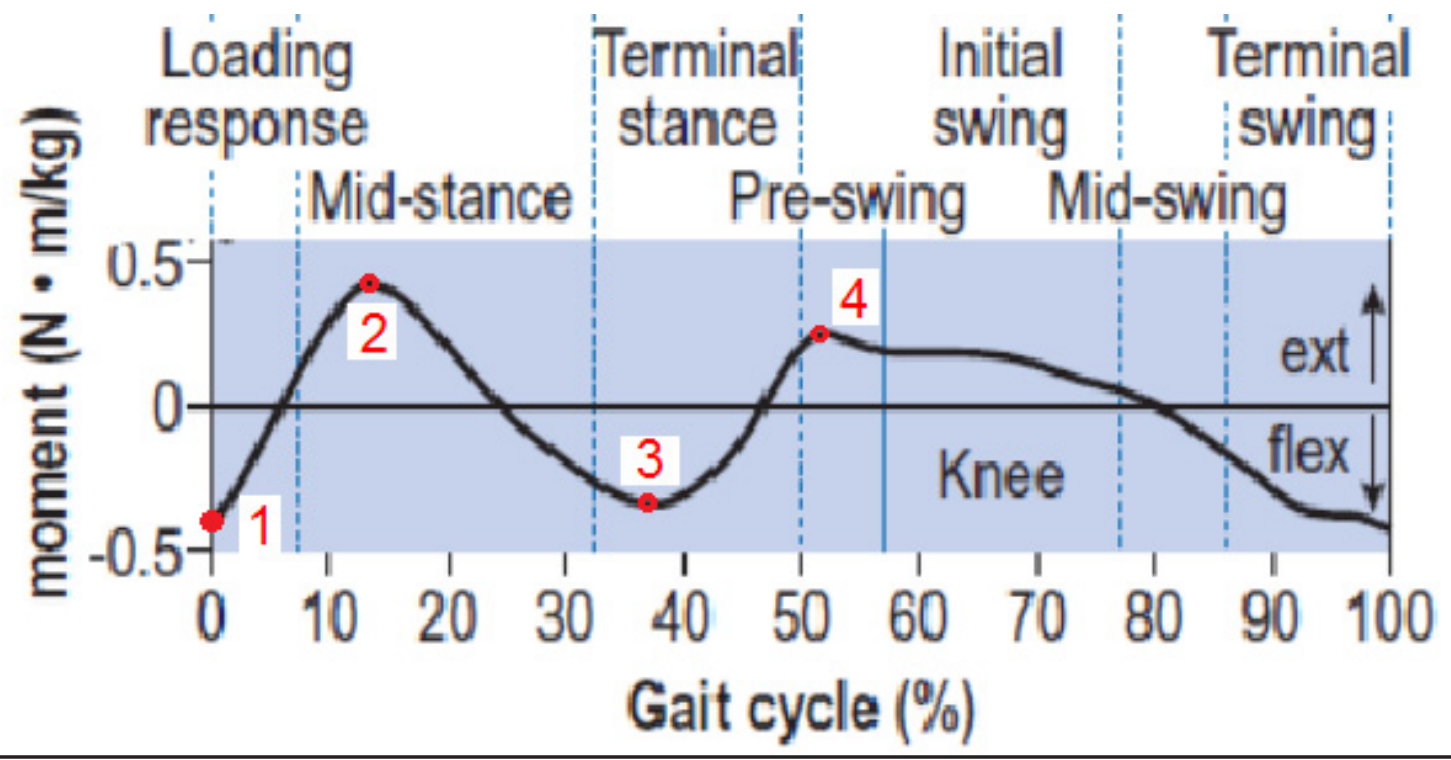

Figura 14. Momentos generados durante la marcha[7].

\begin{tabular}{|c|c|c|c|}
\hline $\begin{array}{c}\text { Puntos de } \\
\text { análisis }\end{array}$ & $\begin{array}{c}\text { Momento } \\
\text { articular }\end{array}$ & $\begin{array}{c}\text { Relación } \\
\text { matemática }\end{array}$ & $\begin{array}{c}\text { Ángulo de } \\
\text { flexión }\end{array}$ \\
\hline Punto 1 & $26 \mathrm{Nm}$ & $0,4 \mathrm{Nm} / \mathrm{kg}$ & $2.86^{\circ}$ \\
\hline Punto 2 & $26 \mathrm{Nm}$ & $0,4 \mathrm{Nm} / \mathrm{kg}$ & $15.71^{\circ}$ \\
\hline Punto 3 & $19.5 \mathrm{Nm}$ & $0,3 \mathrm{Nm} / \mathrm{kg}$ & 0 \\
\hline Punto 4 & $16.25 \mathrm{Nm}$ & $0,25 \mathrm{Nm} / \mathrm{kg}$ & 12.86 \\
\hline
\end{tabular}

Tabla 8. Análsis de momentos articulares.

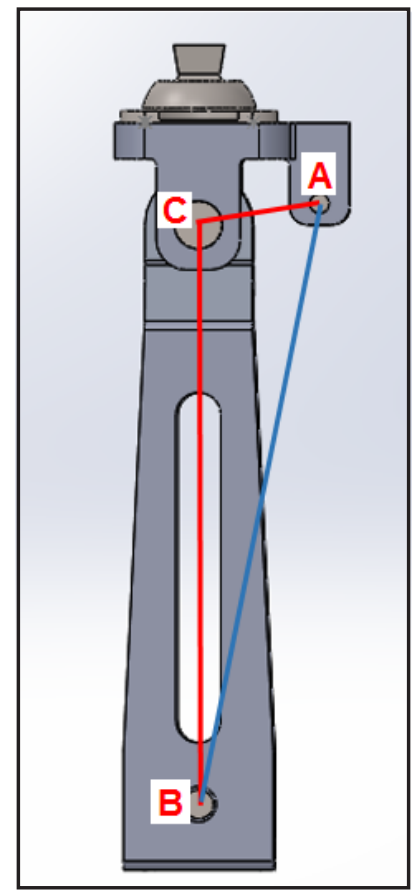

Figura 15. Geometría del modelo. 
diferentes grados de flexión, así entonces el brazo de palanca de aplicación de la fuerza también varía en longitud, como se muestra en la figura 16.

Con la ecuación 1 se determina la fuerza necesaria para el actuador, en donde " $\mathrm{T}$ " corresponde al momento articular de la tabla VIII y "d" la distancia (segmento $\mathrm{CD}$ ) perpendicular a la fuerza de dirección $\mathrm{ADB}$ de acuerdo a la figura 16 y dirigida hacia la articulación de la rodilla ubicada en C.

Los resultados obtenidos se muestran en la tabla IX, para seleccionar el ángulo de flexión con el que se realiza el diseño.

Se realiza el análisis para 4 puntos que corresponden a 4 diferentes grados de flexión o exten- sión de la articulación de la rodilla, debido a que la mayor fuerza para el actuador es para el punto 1 se trabaja para 2.86 grados de flexión.

La velocidad lineal del actuador es dependiente de la velocidad angular de la articulación de la rodilla, se toma en consideración una velocidad de $2,16 \mathrm{Km} / \mathrm{h}$ como velocidad baja de marcha humana, y corresponde a 46 pasos por minuto.

Cada ciclo de marcha contiene la acción de 2 pasos, así entonces 46 pasos corresponde a 23

ciclos de marcha con un tiempo de duración de 2,6 segundos para cada ciclo de marcha.

La Figura 17 muestra la magnitud de la velocidad lineal que debe tener el actuador de acuerdo a un ciclo de marcha con una velocidad de $2,16 \mathrm{~km} / \mathrm{h}$.

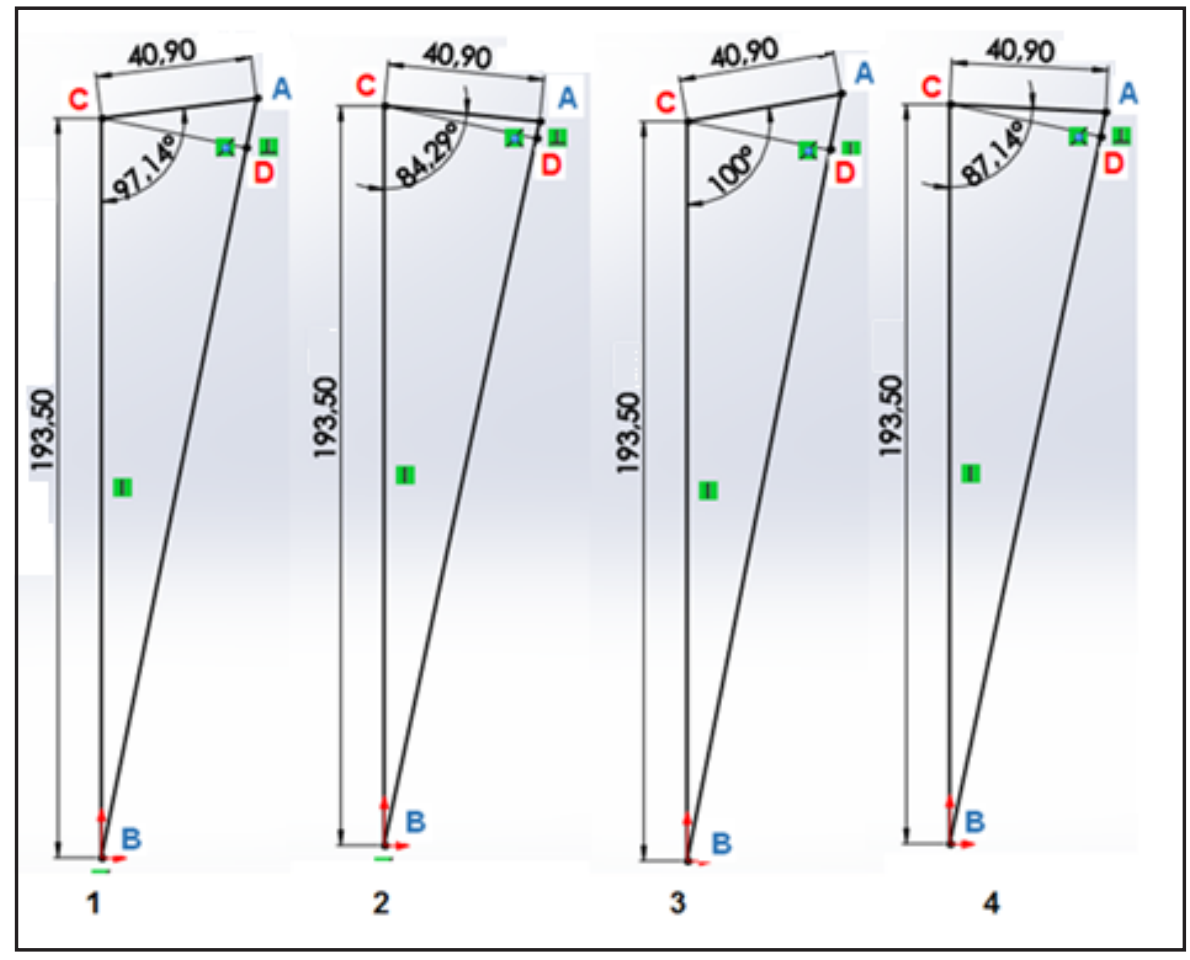

Figura 16. Brazo de palanca para los puntos de análisis 1, 2, 3 y 4.

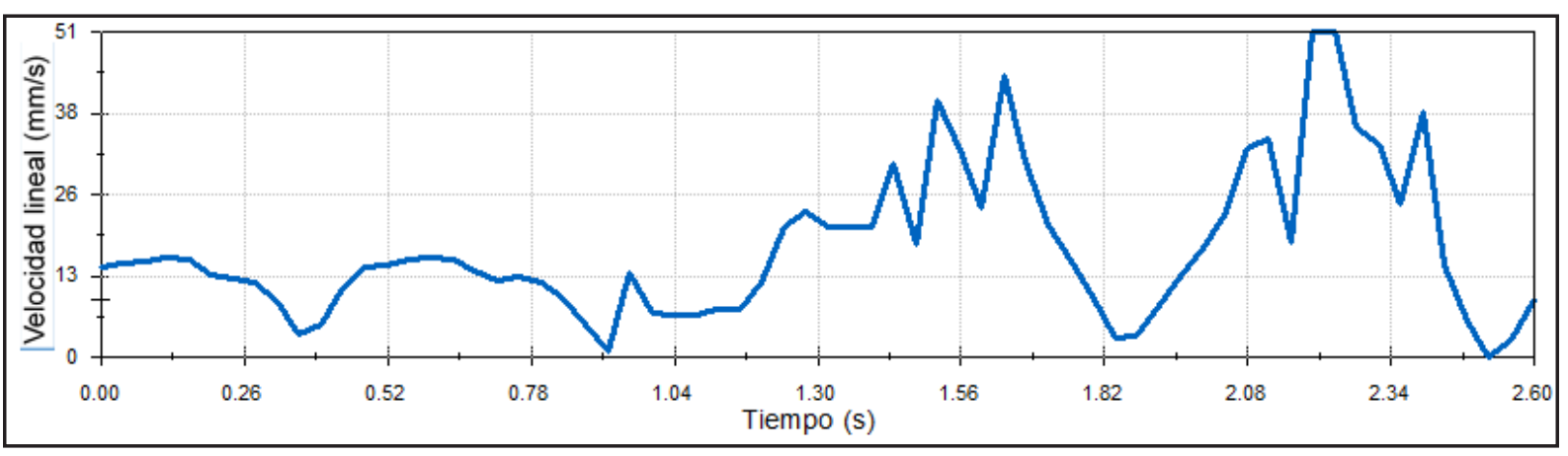

Figura 17. Velocidad lineal de acuerdo al ciclo de marcha. 
La velocidad máxima que debe tener el actuador es $51 \mathrm{~mm} / \mathrm{s}$ y se presenta en el 85\% del ciclo de marcha correspondiente a la terminación de la fase de balanceo, como se muestra en la figura 17.

El actuador para el mecanismo debe ser de accionamiento lineal de acuerdo al modelo de la articulación, debe proporcionar una fuerza de $671,14 \mathrm{~N}$ y una velocidad lineal de $51 \mathrm{~mm} / \mathrm{s}$ como mínimo de acuerdo a los análisis realizados en las secciones anteriores.

El actuador lineal seleccionado cumple con los requerimientos antes mencionados, así entonces las características más importantes se muestran en la tabla X:
Selección de las partes constitutivas

De acuerdo a la fig. 8 las partes diseñadas son la parte móvil y la estrucutra base sin embargo para que el mecanismo sea funcional es indispensable seleccionar componentes que se muestran a continuación.

Los componentes seleccionados son personalizados como el encaje para cada paciente, mientras que los acoples entre la cuenca, rodilla y pie protésico son estándares y realizados en materiales como titanio, acero inoxidable y aluminio y es más facil y económico seleccionarlos. (ver figura 18)

\begin{tabular}{|c|c|c|c|c|}
\hline Punto & Angulo de flexión ( ${ }^{\circ}$ ) & Torque (Nm) & Brazo de palanca & Fuerza (N) \\
\hline Punto 1 & 2.86 & 26 & 38.74 & 671.14 \\
\hline Punto 2 & 15.71 & 26 & 40.64 & 639.76 \\
\hline Punto 3 & 0 & 19.5 & 38.09 & 511.94 \\
\hline Punto 4 & 12.86 & 16.25 & 40.38 & 402.42 \\
\hline
\end{tabular}

Tabla 9. Fuerza del actuador para los puntos críticos de la marcha.

\begin{tabular}{|c|c|}
\hline Características & Detalle \\
\hline Voltaje de entrada & $12 / 24 \mathrm{~V}$ \\
\hline Carrera & 2 pulgadas \\
\hline Fuerza dinámica & $150 \mathrm{lb}$ \\
\hline Fuerza estática & $300 \mathrm{lb}$ \\
\hline Velocidad & 2 pulgadas / Segundo \\
\hline Grado de protección & IP54 \\
\hline Corriente máxima de consume & $5 \mathrm{~A}$ \\
\hline Material constitutive & Aleación de aluminio 6062 \\
\hline
\end{tabular}

Tabla 10. Características del motor eléctrico seleccionado.

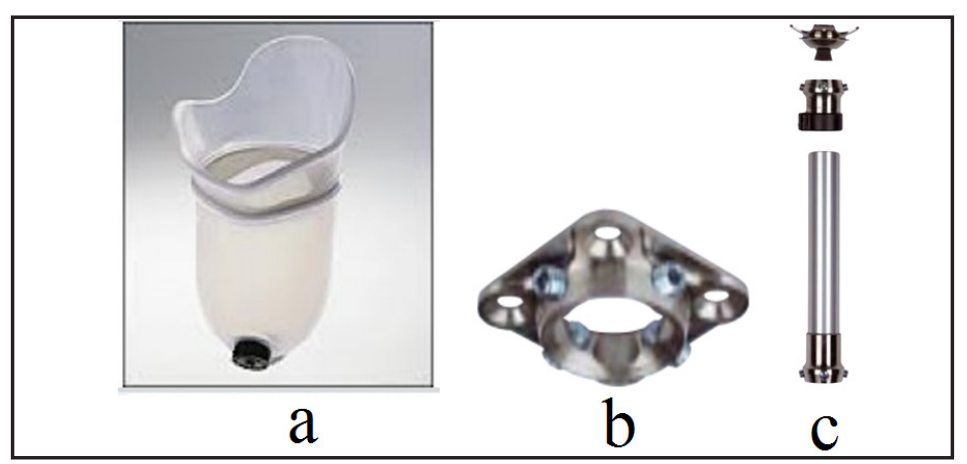

Figura 18. Partes constitutivas a) Cuenca b) Adaptador de Cuenca c) Pilón con abrazadera de tubo. 


\section{Análisis de elementos finitos}

Los componentes mecánicos en forma de vigas, barras simples, etc., se pueden analizar con bastante facilidad por medio de métodos básicos de la mecánica que proporcionan soluciones aproximadas. Sin embargo, los componentes reales rara vez son tan sencillos, y el diseñador se ve forzado a realizar aproximaciones menos eficaces mediante soluciones cercanas, experimentación o métodos numéricos [11].

Los elementos tetraédricos con cuatro nodos en sus vértices son seleccionados para realizar el análisis, ya que se adpata a las geometrías del modelo propuesto por ser elementos para estructuras sólidas. (ver figura 19)

Los materiales seleccionados y adecuados para el mecanismo de acuerdo a la figura 8 se muestran en la tabla XI.

Es necesario restringir los movimientos del mecanismo en relación a sus partes fijas como se muestra en la fig. 20, emulando así la pisada en la fase de apoyo y la reacción que se genera debido al peso del cuerpo de la persona.

A continuación se realiza un mallado de los componentes, y se usa elementos tetraédricos como se muestra en la figura 21.

Para la aplicación de la fuerza 1 (fig. 22), se toma en consideración que la rodilla actúa bajo compresión, entonces se hace uso del valor del peso del cuerpo como la fuerza de compresión aplicada en el mecanismo y es $637 \mathrm{~N}$.

La fig. 23 muestra el momento articular que se genera en la rodilla durante el ciclo de marcha más crítico, la barra ubicada en lugar del actuador (figura 24) transmite el momento articular y parte de la fuerza de compresión.

La distribución de esfuerzos se puede observar en la figura 25a, y las deformaciones unita-

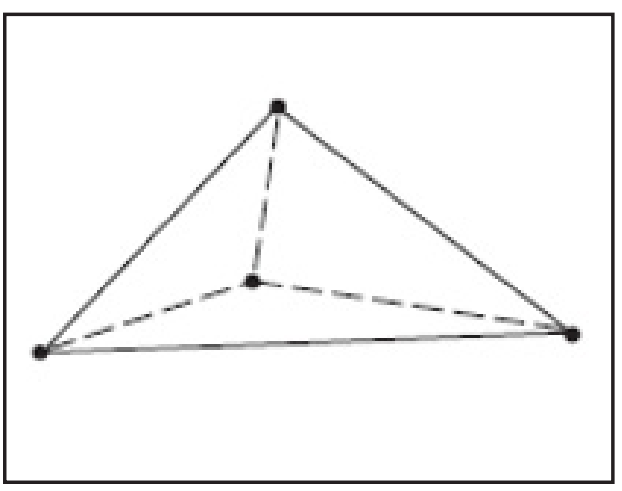

\begin{tabular}{|c|c|}
\hline Parte & Material \\
\hline Móvil & Aleación de aluminio 7075 - T6 \\
\hline Estructura base & Aleación de aluminio 7075 - T6 \\
\hline Pasador 1 & Acero AISI 304 \\
\hline Pasador 2 & Acero AISI 304 \\
\hline Pasador 3 & Acero AISI 304 \\
\hline Acople de pirámide & Aleación de titanio \\
\hline
\end{tabular}

Figura 19 (izquierda). Tetraedro de 4 nodos.

Tabla 11 (derecha). Materiales para el Análisis de elementos finitos.

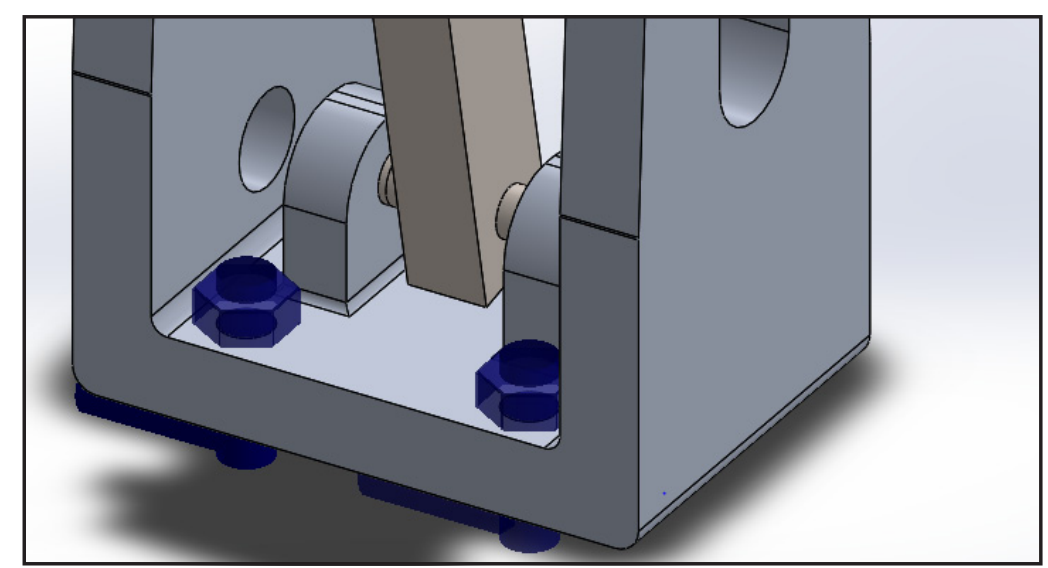

Figura 20. Sujeciones del mecanismo en la parte inferior. 
rias en la gráfica $25 \mathrm{~b}$ y factor de seguridad en la figura 26 para la parte móvil y estructura base ya que son las piezas de diseño.

$\mathrm{Al}$ realizar la simulación del prototipo de rodilla se determinan los esfuerzos combinados de
Von Mises, dicha teoría es la más precisa para materiales dúctiles. El modelo falla cuando el esfuerzo supera el límite elástico del material y es fácilmente comprobable a través del factor de seguridad. El factor de seguridad debe ser por lo

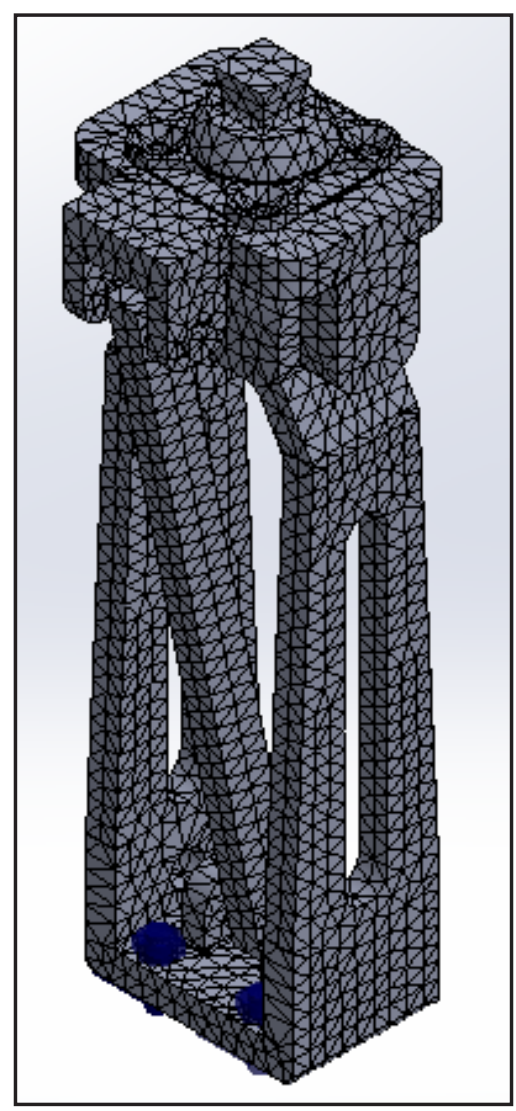

Figura 21. Mallado del mecanismo.

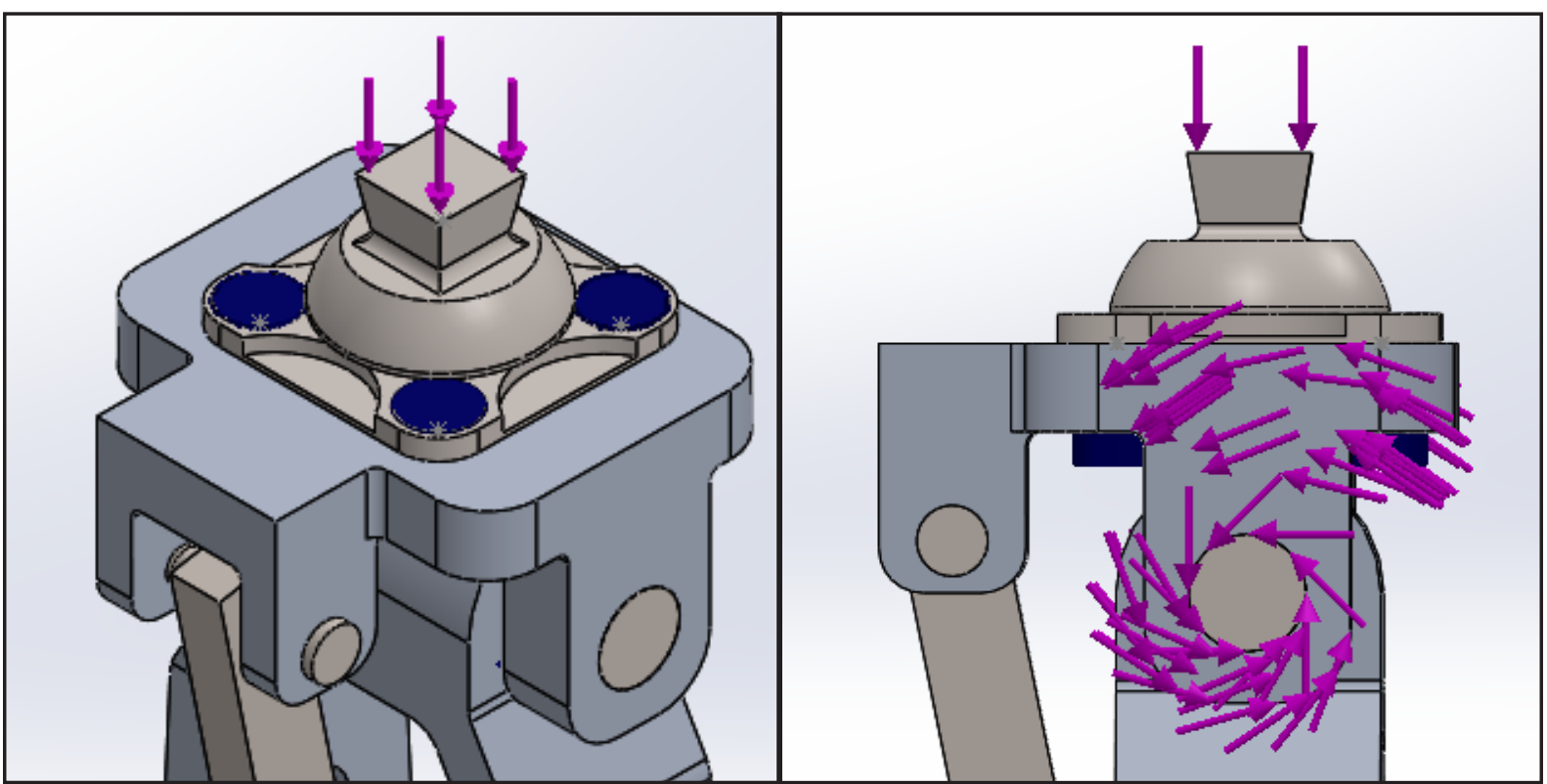

Figura 22 (izquierda). Fuerza de compresión de una persona.

Figura 23 (derecha). Momento articular ejercido durante el ciclo de marcha. 
menos superior a 1 .

Los valores de factor de seguridad reflejan cuantitativamente si el modelo puede fallar bajo las condiciones de contorno a las cuales fue sometido, en el caso en cuestión el factor de seguridad es alto en todo el mecanismo y puede traducirse así mismo que el mecanismo puede soportar una sobrecarga.

No se consideraron fuerzas de impacto tales como tropezar con la prótesis o realizar actividades que correspondan a un nivel de actividad física superior tales como correr o subir escaleras.

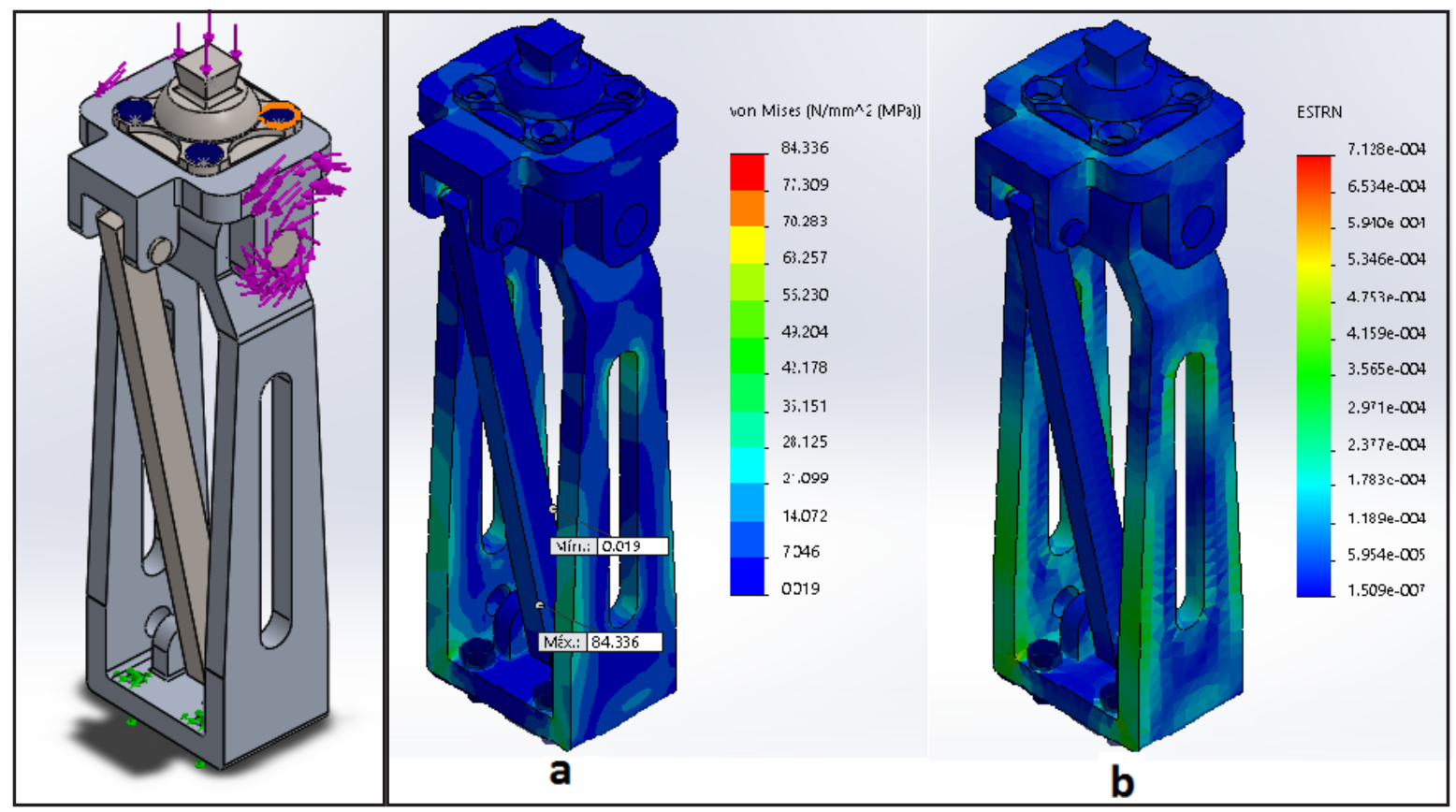

Figura 24 (izquierda). Condiciones de contorno en el mecanismo.

Figura 25 (derecha). Esfuerzos combinados (a) Deformaciones (b).

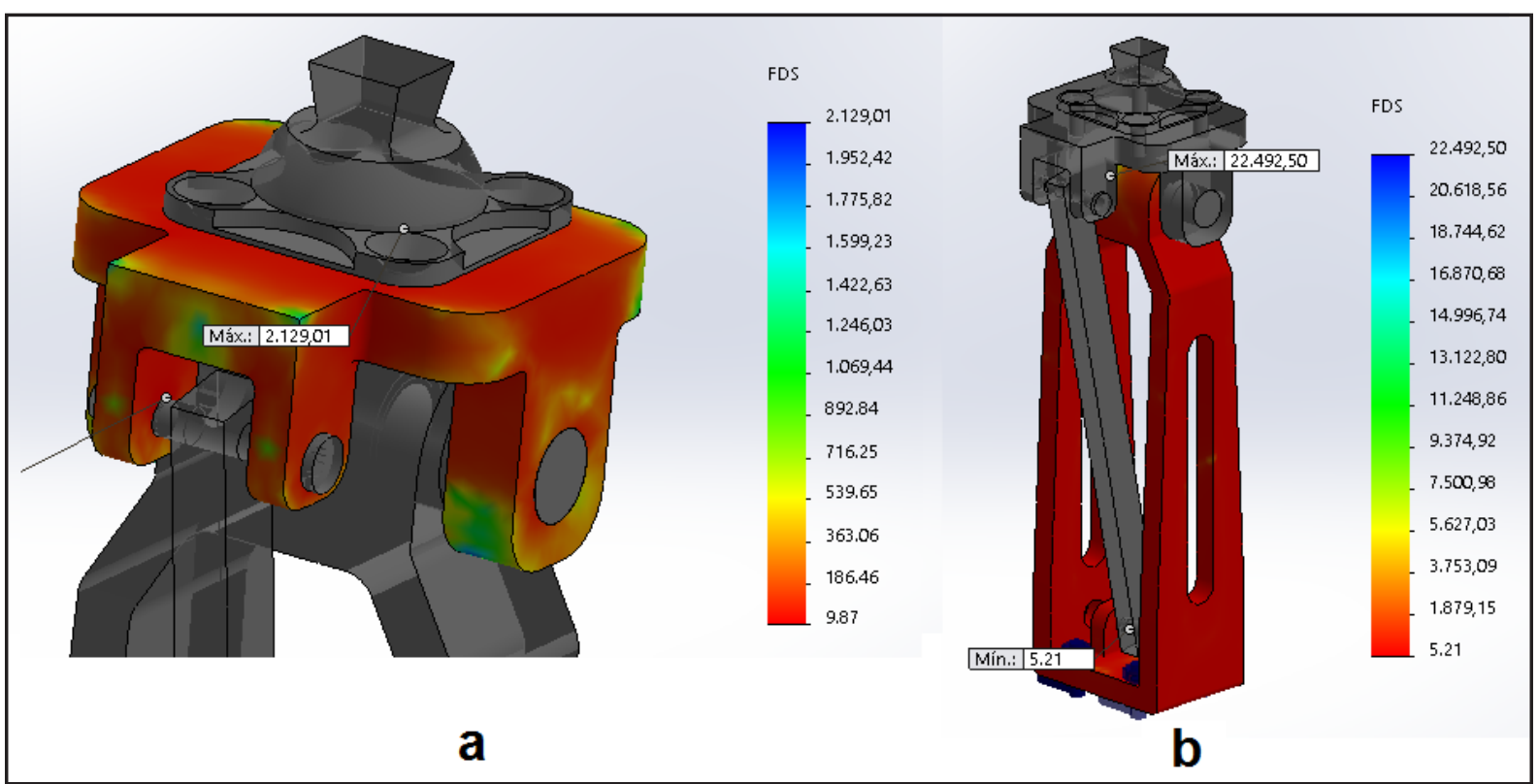

Figura 26. Factor de seguridad a) móvil b) estructura base. 
La figura 27 y figura 28 muestran la distribución de esfuerzos combinados y factor de seguridad respectivamente, en la figura 27 se identifican los puntos más críticos, mientras que en la figura 28 se busca el factor de seguridad para dichos puntos.
El factor de seguridad mínimo es 9.87 ubicado en la unión entre pasado y parte móvil, el factor de seguridad máximo para los puntos críticos es de 45 ubicado en la unión entre el pasador principal de la articulación y la parte móvil.

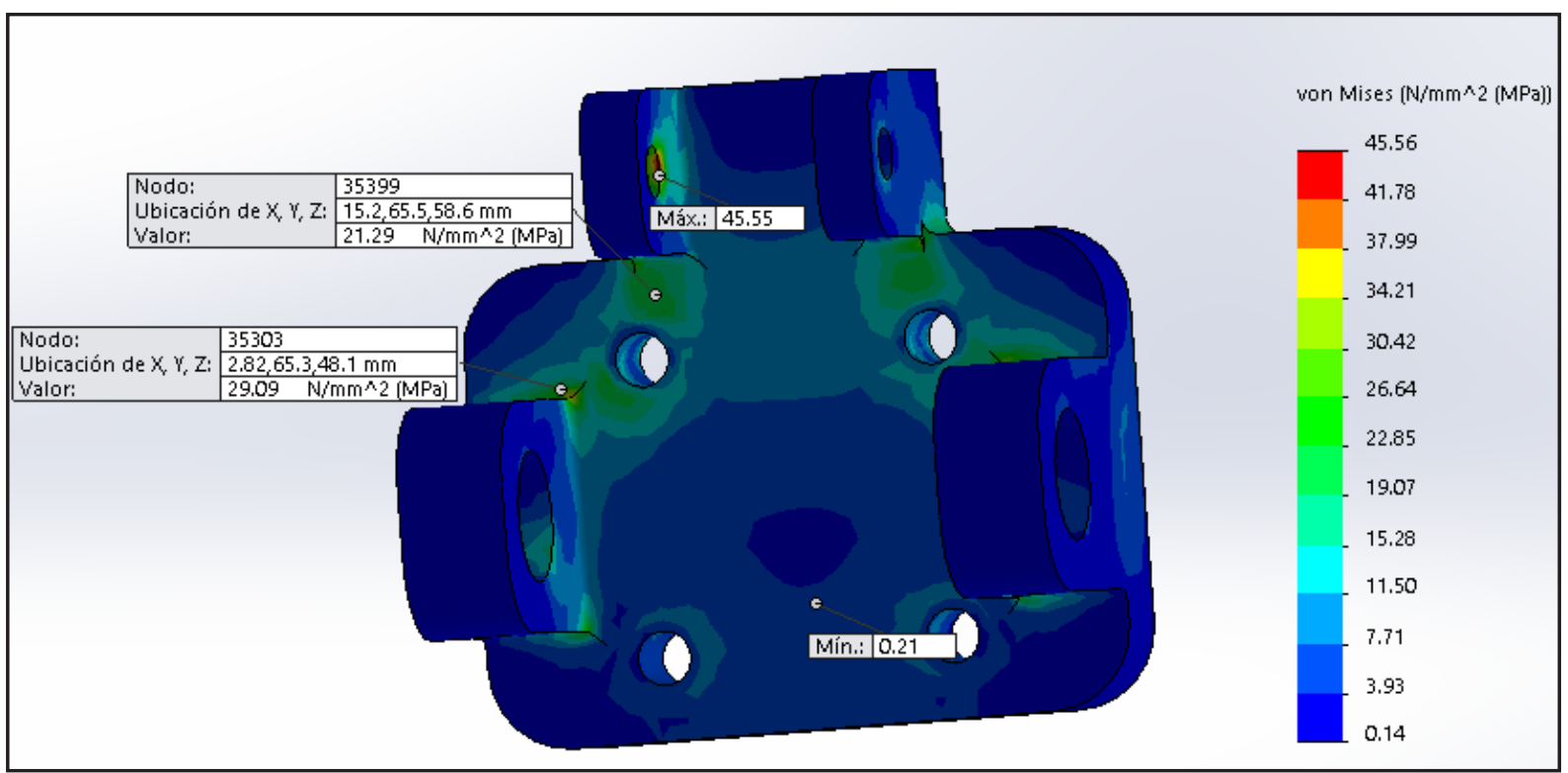

Figura 27. Distribución de esfuerzos de la parte móvil.

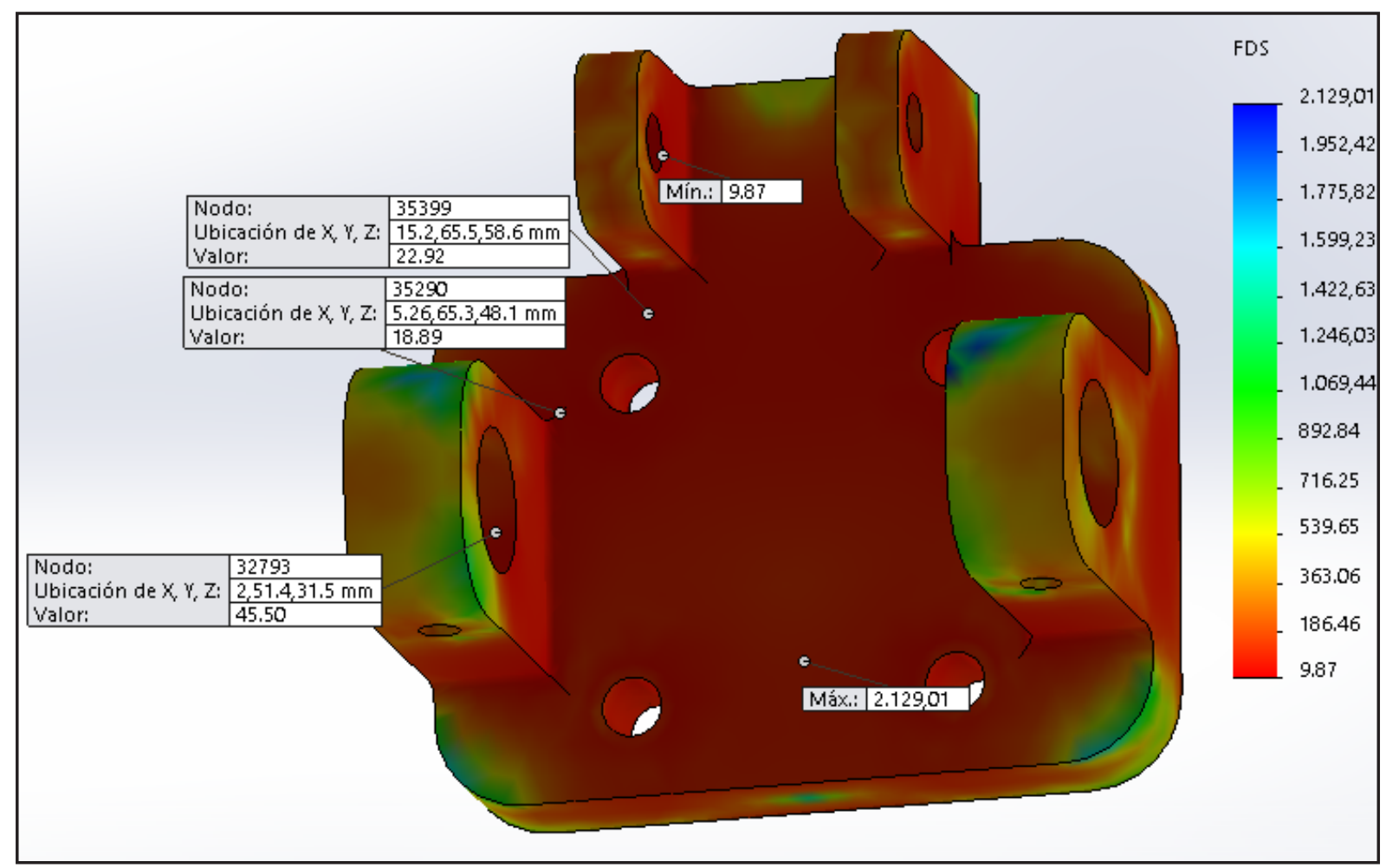

Figura 28. Factor de seguridad para la vista 1. 
En la figura 29 y figura 30 se muestra la distribución de esfuerzos y factor de seguridad para una vista superior de la parte móvil, en la figura 29 se señalan los puntos críticos, mientras en la figura 30 se muestra el factor de seguridad.
En factor de seguridad mínimo es 9.87, mientras que el factor de seguridad máximo para los puntos críticos es 1256, ubicado en la unión entre pasador principal y parte móvil.

Los puntos críticos o de mayor esfuerzo se

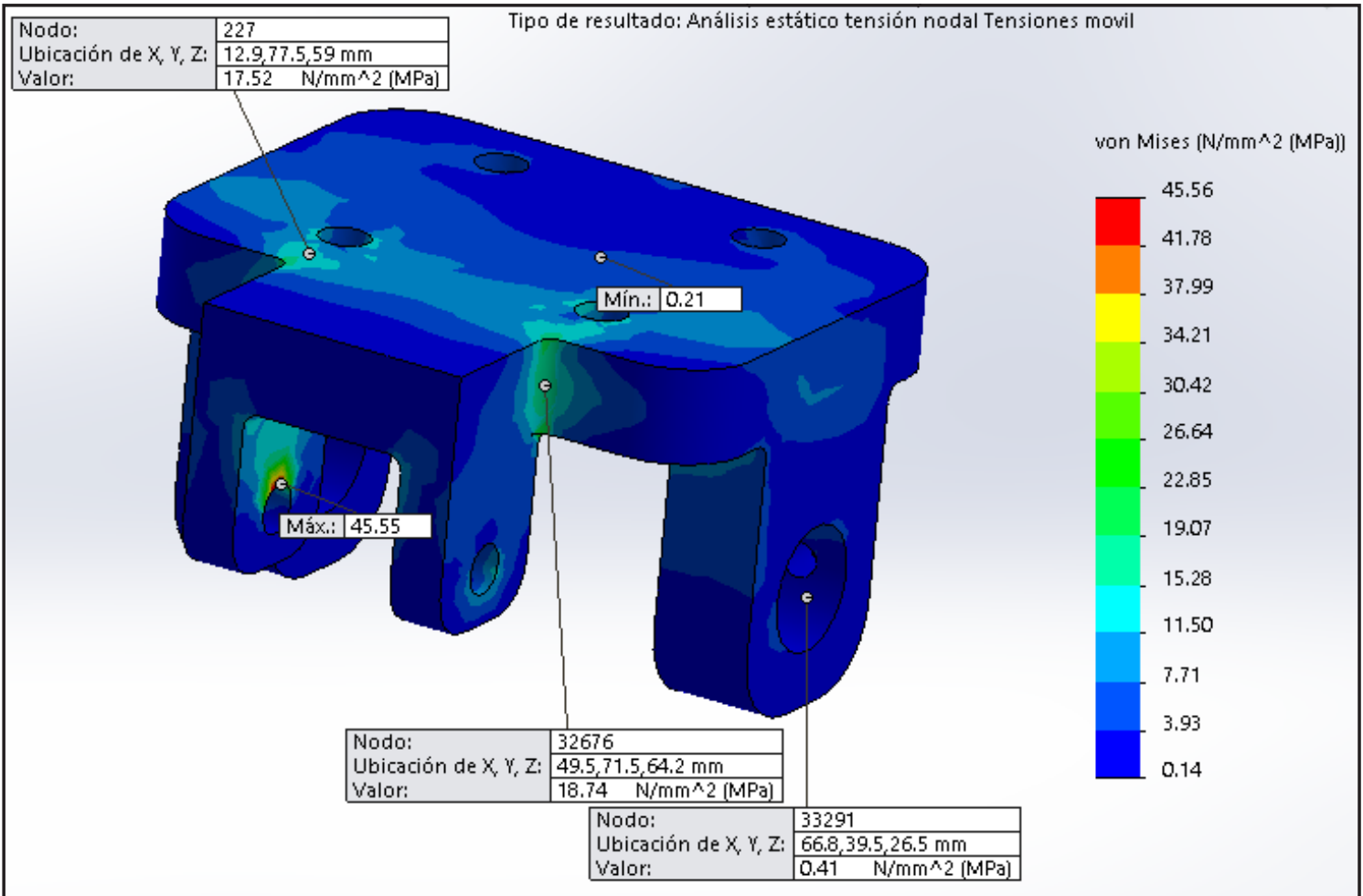

Figura 29. Distribución de esfuerzos de la parte móvil.

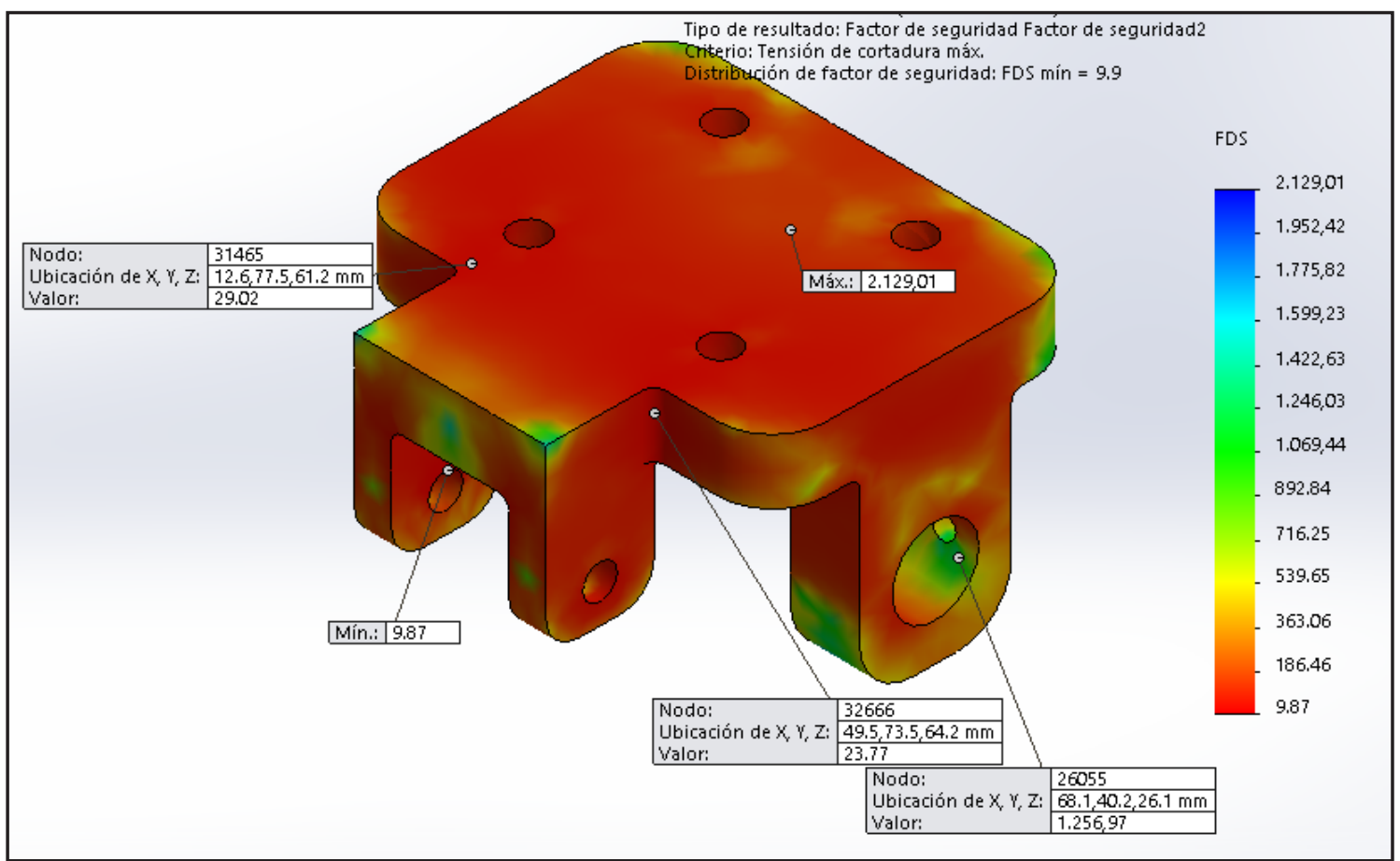

Figura 30. Factor de seguridad para la distribución de esfuerzos de la vista. 
muestran en la figura 31, mientras en la figura 32 se muestra el factor de seguridad para tales puntos. El factor de seguridad mínimo será de 5.1 mientras que el factor de seguridad máximo de los puntos críticos es 53 .
El máximo esfuerzo se puede observar desde la vista posterior únicamente (Fig. 33), el factor de seguridad (Fig. 34) mínimo es 5.21, mientras que el máximo de los puntos críticos es 16 .

Para determinar de un valor de esfuerzo que

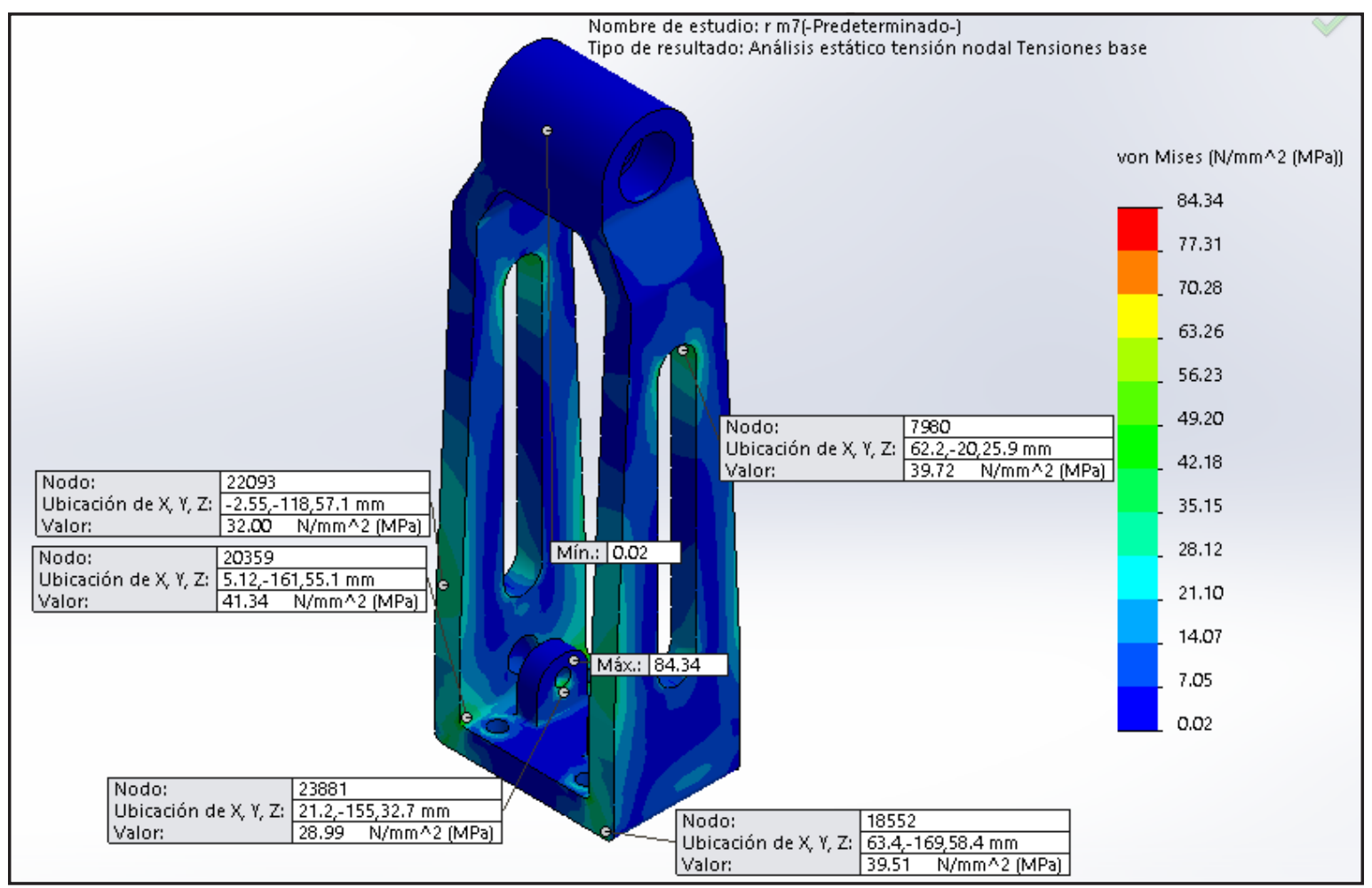

Figura 31. Distribución de esfuerzos de la estructura base vista isométrica.

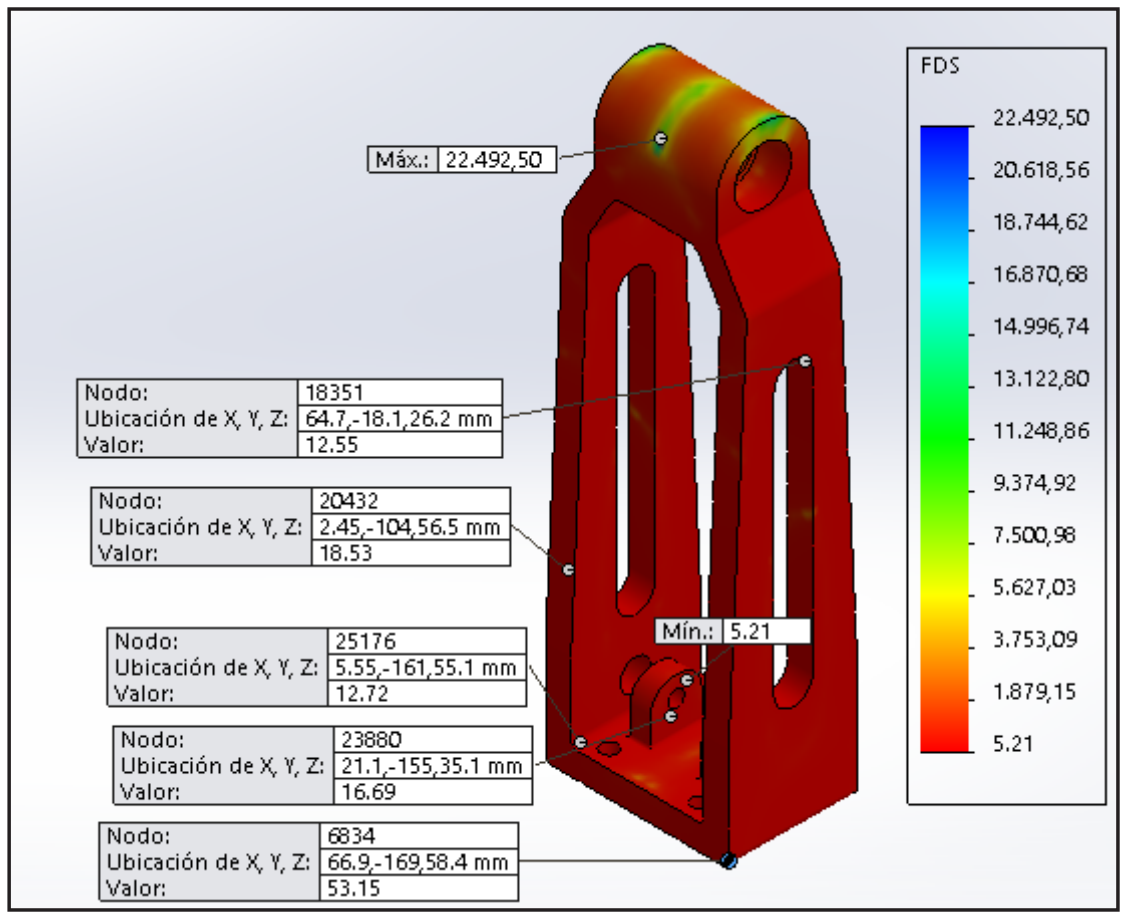

Figura 32. Factor de seguridad para la vista isométrica. 
sea adecuado, se realiza gráficas de Esfuerzo vs número de elementos que se muestran en la fig. 35 y fig. 36.

Un mallado óptimo es aquel que permite obtener un resultado aproximado al real en con- junción con un rápido cálculo computacional [12]. Para tal fin se hace un análisis de sensibilidad de esfuerzos de Von Misses como se muestra en la figura 35 y figura 36.

Se puede observar en la tabla XII que a partir

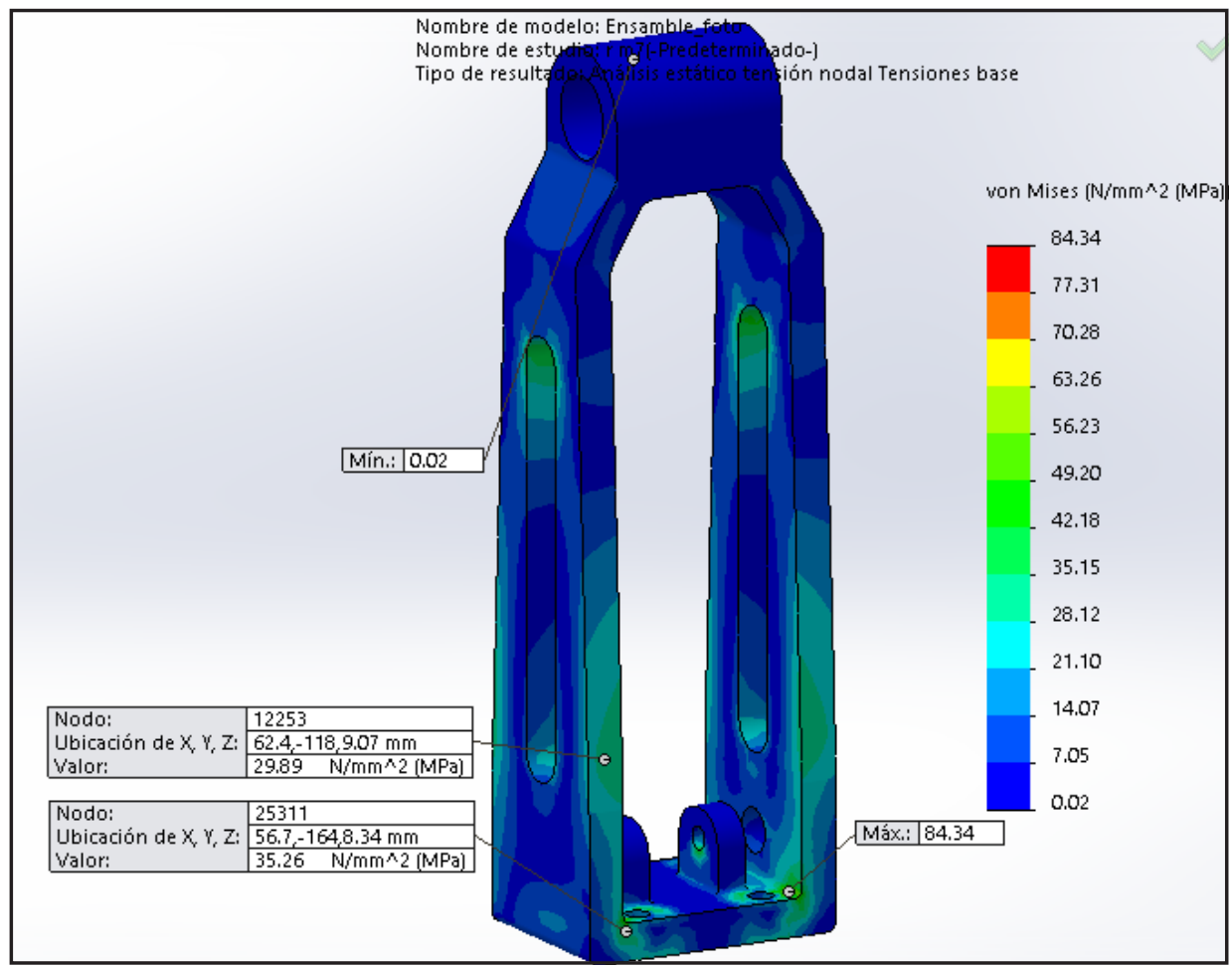

Figura 33. Distribución de esfuerzos de la estructura base vista posterior.

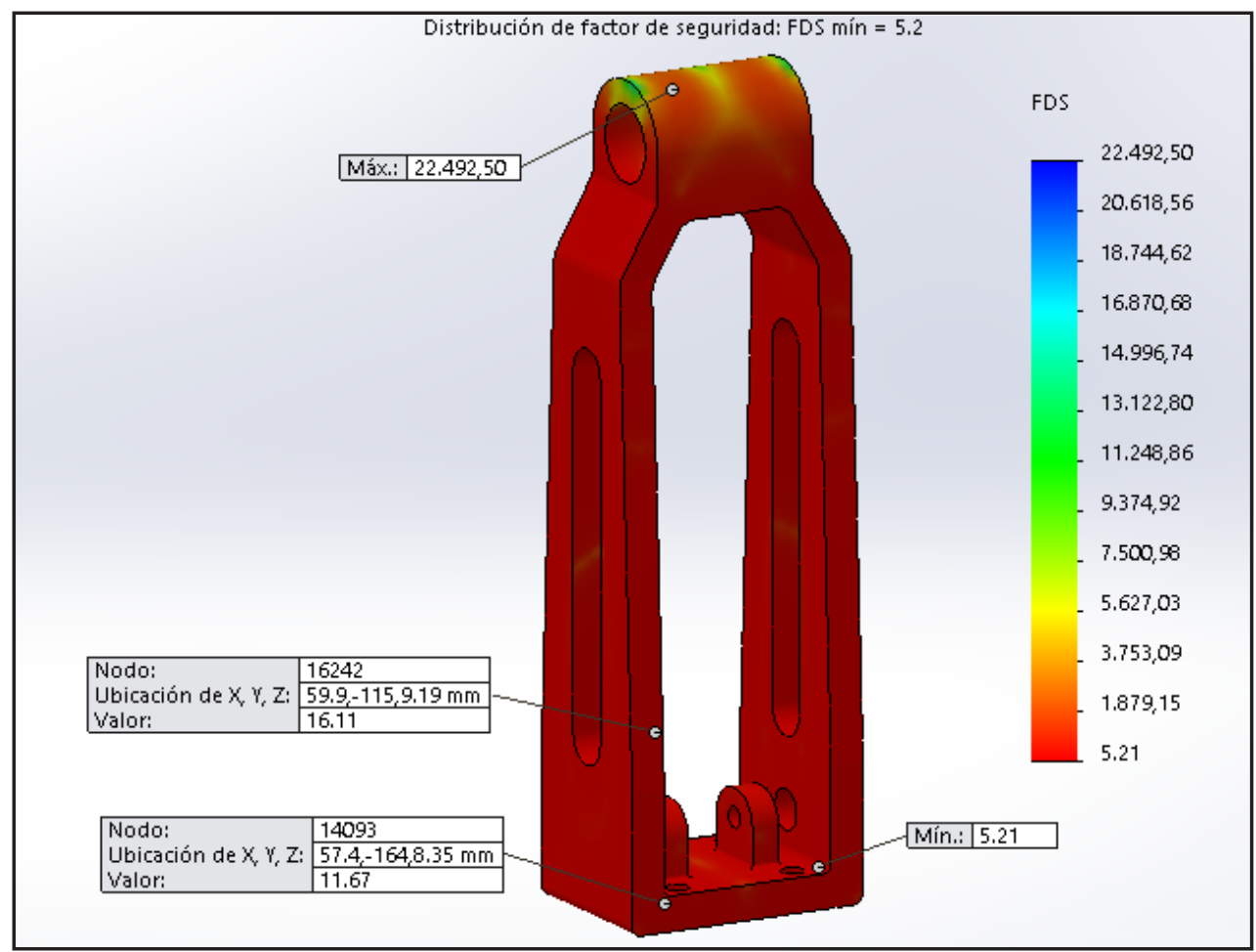

Figura 34. Factor de seguridad de la estructura base vista posterior. 
de 26104 elementos el error porcentual es menor al 5\%, los datos que se usan para el análisis son los del mallado 7 contiene: 31626 elementos, elementos de $5,65 \mathrm{~mm}$ con una tolerancia de $0,28 \mathrm{~mm}$ y 38400 nodos.

Conviene a menudo, en situaciones que implican esfuerzos de tensión y cortante combinados que actúan sobre un mismo punto, definir un esfuerzo efectivo que sirva para represen- tar la combinación de esfuerzos. El enfoque de la energía de distorsión proporciona un buen medio para hacer esto en materiales dúctiles. [8]

El factor de seguridad para materiales dúctiles bajo carga estática puede calcularse mediante la ecuación 5, Sy corresponde al límite de fluencia del material, mientras que $\sigma_{\mathrm{VM}}$ corresponde al esfuerzo de Von Misses.

\section{Esfuerzo - Número de elementos}

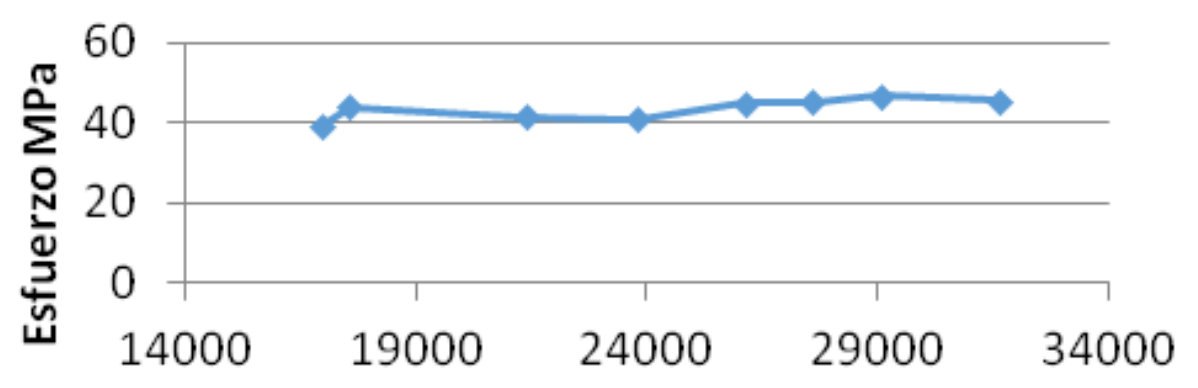

Número de elementos

Figura 35. Gráfica esfuerzo - número de elementos de la parte móvil.

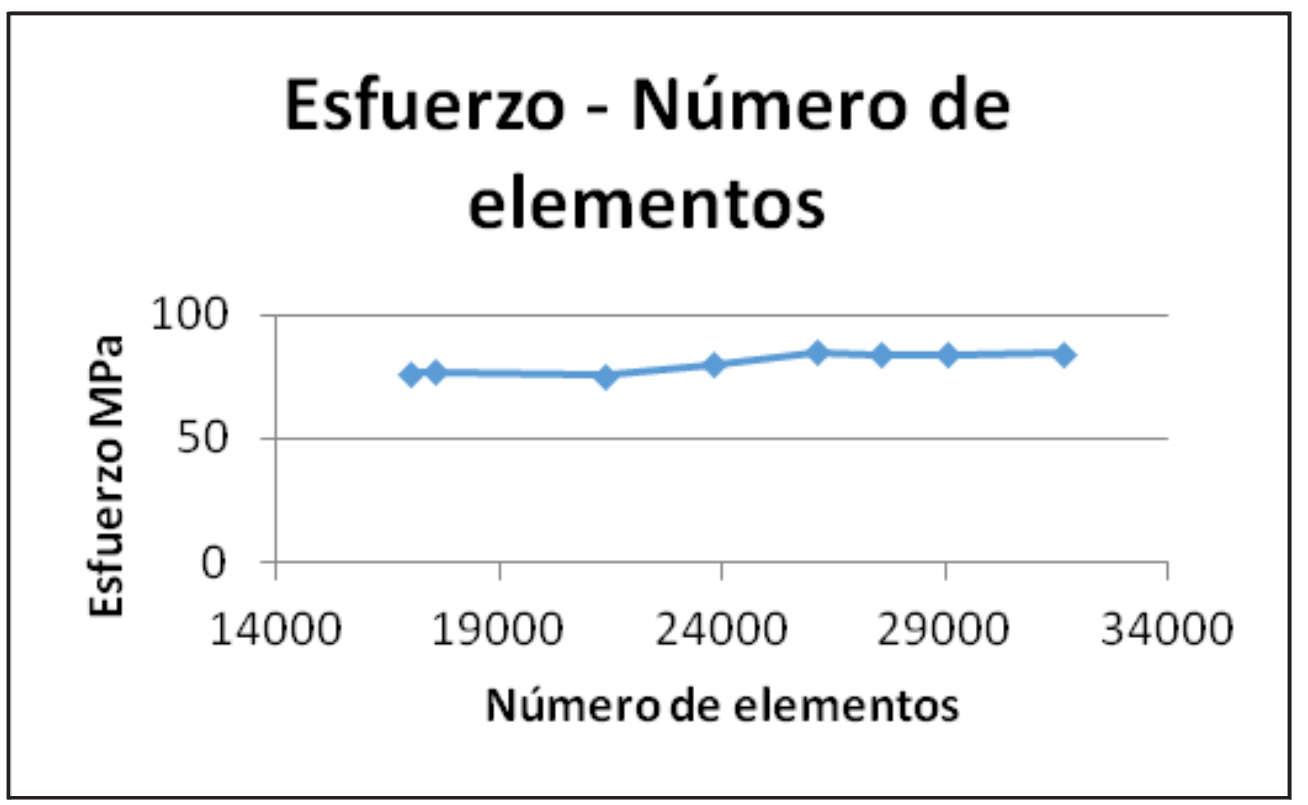

Figura 36. Gráfica esfuerzo - número de elementos de la estructura base. 
Ecuación 5: Factor de seguridad según la teoría de energía de la distorsión[8]

$$
\mathrm{N}=\mathrm{Sy} / \sigma \mathrm{VM}
$$

\section{Prototipado}

El uso de un polvo cerámico como material para el prototipo de prótesis nos facilita realizar pruebas dinámicas, y empezar a trabajar con un modelo físico. (ver tabla XIII)

El prototipo impreso con polvo cerámico se muestra en la figura 37, puesto que este prototipo no es lo suficiente funcional y tampoco tiene la resistencia necesaria, solo se usa para tener una mejor apreciación del diseño y realizar pequeños cambios para mejorar el modelo.

Se usa fresadora CNC de 4 ejes ROMI D 1250 y una cortadora por electro hilo Chmer en la construcción del mecanismo en aluminio 7075, obteniendo los resultados que se muestran en la figura 38.

Los elementos constitutivos son proporcionados por la fundación "Prótesis para la vida" ubicada en el Ecuador y son totalmente compatibles con el modelo propuesto, se muestra en la figura 39.

\begin{tabular}{|c|c|c|c|c|c|}
\hline Malla & $\begin{array}{c}\text { Nro. } \\
\text { Elementos }\end{array}$ & $\begin{array}{c}\text { Esfuerzos } \\
\text { combinados } \\
\mathbf{( M P a})\end{array}$ & $\begin{array}{c}\text { Esfuerzos } \\
\text { combinados } \\
\mathbf{( M P a )}\end{array}$ & $\begin{array}{c}\text { Error } \\
\text { porcentual } \\
\text { (parte móvil) }\end{array}$ & $\begin{array}{c}\text { Error porcentual } \\
\text { (estructura base) }\end{array}$ \\
\hline $\mathbf{0}$ & 16982 & 39,26 & 76,33 & & \\
\hline $\mathbf{1}$ & 17577 & 43,92 & 76,84 & 11,87 & 0,67 \\
\hline $\mathbf{2}$ & 21385 & 41,49 & 75,79 & 5,53 & 1,37 \\
\hline $\mathbf{3}$ & 23779 & 41,04 & 79,83 & 1,08 & 5,33 \\
\hline $\mathbf{4}$ & 26104 & 44,93 & 85,26 & 9,48 & 6,80 \\
\hline $\mathbf{5}$ & 27572 & 45,12 & 84,16 & 0,42 & 1,29 \\
\hline $\mathbf{6}$ & 29055 & 46,92 & 83,94 & 3,99 & 0,26 \\
\hline $\mathbf{7}$ & 31626 & 45,55 & 84,34 & 2,92 & 0,48 \\
\hline
\end{tabular}

Tabla 12. Datos de esfuerzo.

\begin{tabular}{|c|c|c|}
\hline Infiltrant & Color bond & Salt Water Cure \\
\hline Composition & VisiJet "PXL" & VisiJet "PXL" \\
\hline Tensile Strength & 14.2 & 2.38 \\
\hline Elongation at break, \% & 0.23 & 0.04 \\
\hline Modulus of Elasticity, Mpa & 9.450 & 12.855 \\
\hline Flexural Strenght & 31.1 & 13.1 \\
\hline Flexural Modulus & 7.163 & 6.355 \\
\hline
\end{tabular}

Tabla 13. Tabla de propiedades para el tratamiento del polvo cerámico[13]. 


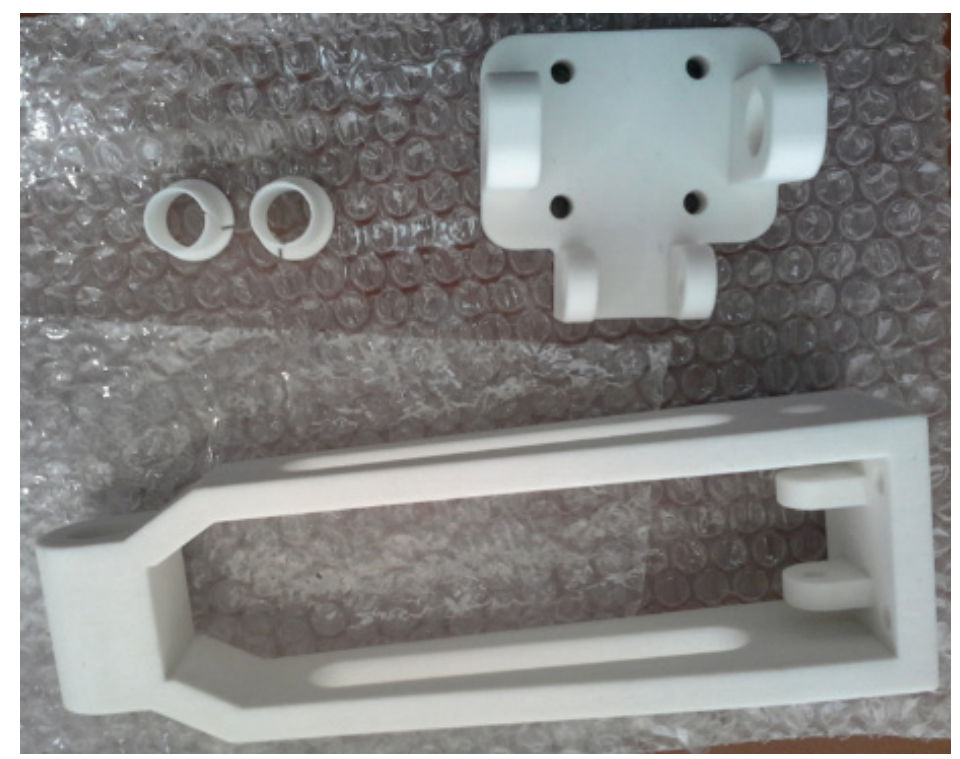

Figura 37. Prototipo de rodilla impreso en 3d.

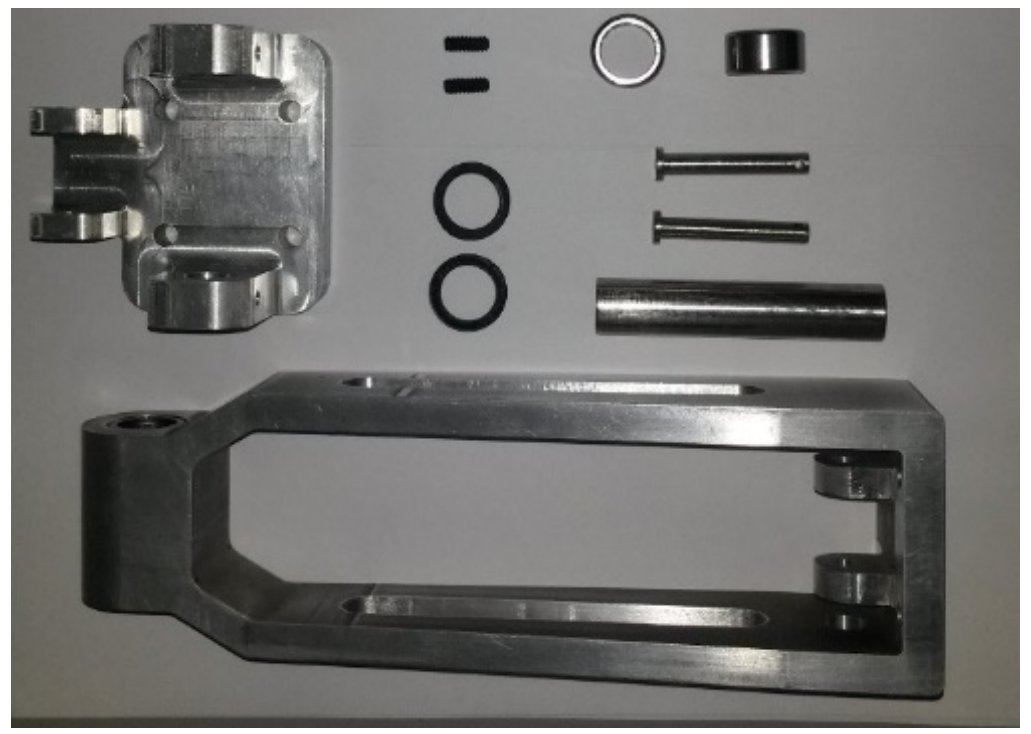

Figura 38. Partes del mecanismo.

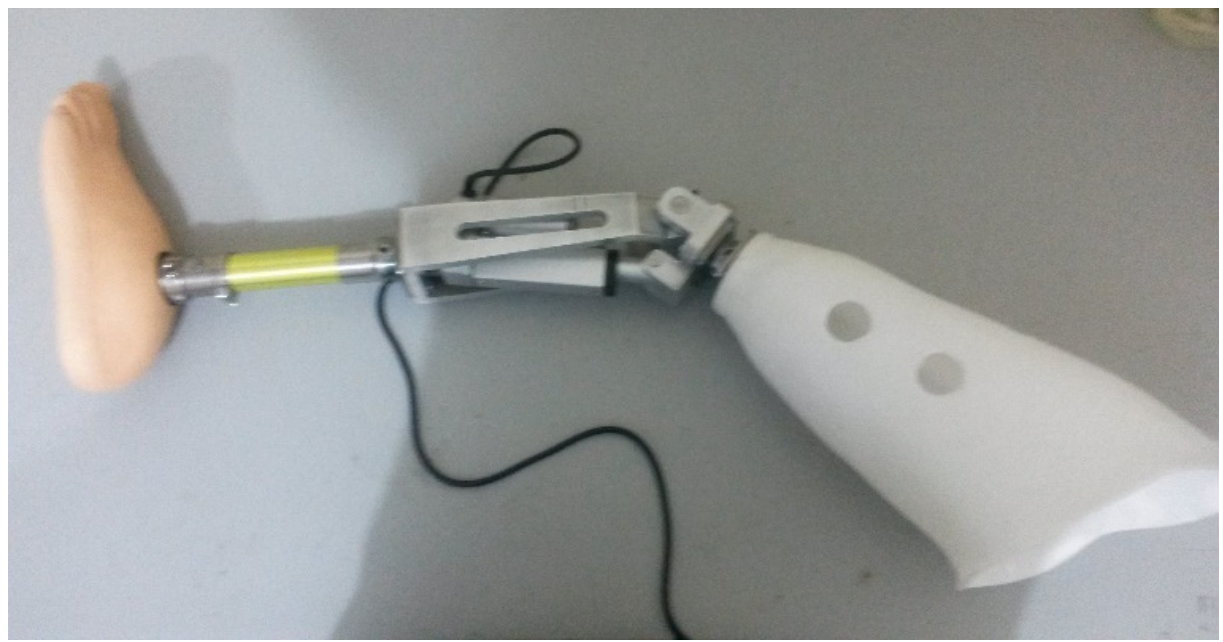

Figura 39. Mecanismo con sus partes. 


\section{Conclusiones}

Las aleaciones de aluminio son el mejor material para la construcción del prototipo por sus propiedades mecánicas, la aleación de aluminio 7075 es el material que se encuentra en nuestro medio con las mejores condiciones de mecanizado y calidad.

Se ha construido un mecanismo de articulación que emula los movimientos de flexo-extensión en 80 grados funcionales.

Se puede diseñar un prototipo de rodilla que emule el funcionamiento de la rodilla humana y satisfaga en gran medida, las exigencias de realizar una marcha humana.

Es posible seleccionar más de un actuador de bajo peso y con dimensiones adecuadas, capaz de proporcionar la fuerza motora necesaria en la caminata humana.

Las herramientas CAD CAE y CAM permiten realizar diseños, predecir fallas durante su funcionamiento y materializarlos.

Los modelos en impresiones $3 \mathrm{~d}$ ayudan a tener una percepción mejor del diseño en físico, para poder realizar pequeñas modificaciones.

\section{Bibliografía}

1. Gobierno de Ecuador, Consejo Nacional de Discapacidades. Causas de discapacidad, 2013.

Au SK, Herr HM. Powered ankle-foot prosthesis. IEEE Robotics \& Automation Magazine 2008;15(3)52-59.

2. Kapandji AI. Fisiología articular ( $6^{\mathrm{a}}$ ed), Editorial Medica Panamericana, Madrid, 2006.

3. Torrealba R, Fernández-López G, Grieco JC. Towards the development of knee prostheses: review of current researches. Kybernetes: The International Journal of Systems \& Cybernetics 2008;37:15611576.

4. Murray MP, Drought AB, Kory RC. Walking patterns of normal men. J. Bone Joint Surg 1964;46A:335-360.

5. Huston R. Principes of Biomecanics. Taylor \& Francis, 2009.

6. Whittle MW. Gait Analysis, an Introduction. Elsevier, New York, 2007. Vol. 4, p.258.

7. Norton RL. Diseños de máquinas. Pearson, México DF, 2011

8. Vaughan C, Davis B, O'Connor J. Dynamics of Humain Gait. Kiboho, South Africa, 1992.

9. Nordin M, Frankel V. Biomecánica básica del sistema músculo esquelético. Fareso S.A., Barcelona, 2001.

10. Shigley JE. Diseño en Ingeniería mecánica. McGraw-Hill/Interamericana Editores, S.A., México, D.F., 2008.

11. Zienkiewicz OC, Taylor RL. The Finite Element Method (4th ed), McGraw-Hill, 1989.

12. 3DSYSTEMS, ProJet $x 60$ Series Professional 3D Printers, USA, 2015 